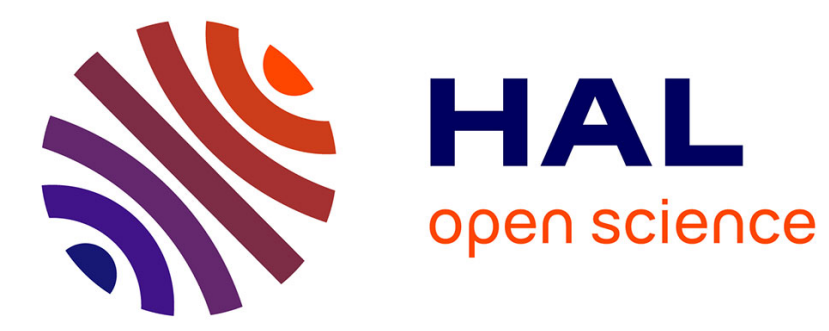

\title{
Microprocessor dynamics and interactions at endogenous imprinted C19MC microRNA genes.
}

Clément Bellemer, Marie-Line Bortolin-Cavaillé, Ute Schmidt, Stig Mølgaard Rask Jensen, Jørgen Kjems, Edouard Bertrand, Jérôme Cavaillé

\section{To cite this version:}

Clément Bellemer, Marie-Line Bortolin-Cavaillé, Ute Schmidt, Stig Mølgaard Rask Jensen, Jørgen Kjems, et al.. Microprocessor dynamics and interactions at endogenous imprinted C19MC microRNA genes.. Journal of Cell Science, 2012, pp.2709-2720. 10.1242/jcs.100354 . hal-00688362

\section{HAL Id: hal-00688362 https://hal.science/hal-00688362}

Submitted on 1 Jun 2021

HAL is a multi-disciplinary open access archive for the deposit and dissemination of scientific research documents, whether they are published or not. The documents may come from teaching and research institutions in France or abroad, or from public or private research centers.
L'archive ouverte pluridisciplinaire HAL, est destinée au dépôt et à la diffusion de documents scientifiques de niveau recherche, publiés ou non, émanant des établissements d'enseignement et de recherche français ou étrangers, des laboratoires publics ou privés. 


\title{
Microprocessor dynamics and interactions at endogenous imprinted C19MC microRNA genes
}

\author{
Clément Bellemer ${ }^{1,2}$, Marie-Line Bortolin-Cavaillé ${ }^{1,2}$, Ute Schmidt ${ }^{3}$, Stig Mølgaard Rask Jensen ${ }^{4, \star}$, \\ Jørgen Kjems ${ }^{4}$, Edouard Bertrand ${ }^{3}$ and Jérôme Cavaillé ${ }^{1,2, \ddagger}$
}

${ }^{1}$ Laboratoire de Biologie Moléculaire Eucaryote (LBME), Université Paul Sabatier (UPS), Université de Toulouse, 31000 Toulouse, France

${ }^{2} \mathrm{CNRS}$, LBME, 31000 Toulouse, France

${ }^{3}$ Institute of Molecular Genetics of Montpellier, CNRS UMR 5535, 34293 Montpellier, France

${ }^{4}$ Interdisciplinary Nanoscience Center iNANO, Department of Molecular Biology, Aarhus University, C.F. Møllers Allé, Building 1130, Room 404, 8000 Aarhus, Denmark

*Present address: National Cancer Institute, Frederick Building 567, Room 261/275, Frederick, MD 21702, USA

${ }^{\ddagger}$ Author for correspondence (cavaille@ibcg.biotoul.fr)

Accepted 2 February 2012

Journal of Cell Science 125, 2709-2720

(C) 2012. Published by The Company of Biologists Ltd

doi: $10.1242 /$ jcs. 100354

\section{Summary}

Nuclear primary microRNA (pri-miRNA) processing catalyzed by the DGCR8-Drosha (Microprocessor) complex is highly regulated. Little is known, however, about how microRNA biogenesis is spatially organized within the mammalian nucleus. Here, we image for the first time, in living cells and at the level of a single microRNA cluster, the intranuclear distribution of untagged, endogenously-expressed pri-miRNAs generated at the human imprinted chromosome 19 microRNA cluster (C19MC), from the environment of transcription sites to single molecules of fully released DGCR8-bound pri-miRNAs dispersed throughout the nucleoplasm. We report that a large fraction of Microprocessor concentrates onto unspliced C19MC pri-miRNA deposited in close proximity to their genes. Our live-cell imaging studies provide direct visual evidence that DGCR8 and Drosha are targeted post-transcriptionally to C19MC pri-miRNAs as a preformed complex but dissociate separately. These dynamics support the view that, upon pri-miRNA loading and most probably concomitantly with Drosha-mediated cleavages, Microprocessor undergoes conformational changes that trigger the release of Drosha while DGCR8 remains stably bound to pri-miRNA.

Key words: Intranuclear RNA trafficking, Transcription sites, microRNA, Nuclear architecture, pri-miRNA processing, DGCR8-Drosha (Microprocessor) complex

\section{Introduction}

MicroRNAs (miRNAs) are tiny ( $22 \mathrm{nt}$ in length) non-proteincoding RNAs that trigger gene silencing at the post-transcriptional level, mostly as a result of imperfect base-pairing with $3^{\prime}$ untranslated regions of cellular mRNAs. miRNA-mediated gene regulation has been shown to play important roles in a myriad of cellular and developmental processes and in disease (Bushati and Cohen, 2007). It is therefore of crucial importance to better understand the molecular mechanisms that underlie regulation of miRNA expression in normal and pathological contexts.

In animals, numerous miRNA loci are embedded within introns of either protein-coding or non-coding RNA genes (Kim et al., 2009). miRNA biogenesis initiates in the nucleus, where long RNA polymerase II (Pol II) primary transcripts (pri-miRNAs) are converted into $\sim 65 \mathrm{nt}$ hairpin-shaped intermediates (premiRNAs) by the Microprocessor complex formed by the RNAse-III-like enzyme Drosha and the double-stranded RNAbinding protein DGCR8. Following their export to the cytoplasm by exportin-5, pre-miRNAs are further processed by Dicer into $\sim 22 \mathrm{nt}$ mature miRNAs that concomitantly associate with specific proteins, particularly Argonaute (Ago) proteins, to form metabolically stable ribonucleoparticles named miRISC (miRNA-induced silencing complex). Through mechanisms still debated, the sequence-specific binding of miRISC promotes gene silencing of the targeted mRNAs, either by blocking translation and/or by accelerating degradation (Filipowicz et al., 2008).

Microprocessor-mediated nuclear processing of pri-miRNA represents an important aspect of the expanding biology of miRNAs, not only because it delineates the functional $5^{\prime}$ end of a subset of miRNAs, the so-called seed sequence that plays a pivotal role in mRNA targeting (Han et al., 2006), but also because the activity of Microprocessor is subject to important post-transcriptional regulation in normal and pathological situations. Indeed, many studies have identified several general RNA-binding proteins that function either as negative or positive regulators of Drosha cropping activities at a sub-set of specific pri-miRNAs, most probably through their ability to control primiRNA folding, recruitment of Microprocessor to pri-miRNAs, and/or endonucleolytic cleavage activity or specificity (Davis et al., 2008; Guil and Cáceres, 2007; Michlewski et al., 2008; Pawlicki and Steitz, 2008; Siomi and Siomi, 2010; Suzuki et al., 2009; Thomson et al., 2006; Viswanathan et al., 2008; Yamagata et al., 2009).

Unraveling the Microprocessor mode of action has made use of reconstituted assays or epitope-tagged proteins (Denli et al., 2004; Faller et al., 2007; Gregory et al., 2004; Han et al., 2004; Han et al., 2006; Landthaler et al., 2004; Yeom et al., 2006). Interestingly, only DGCR8 interacts with pri-miRNAs. It binds 
the junction between the double-stranded stem and the flanking single-stranded RNA sequences, acting as 'a molecular ruler' to correctly position the processing center of Drosha $\sim 11 \mathrm{bp}$ from the junction (Han et al., 2006; Lee et al., 2006; Yeom et al., 2006). How endogenously-expressed Microprocessor recognizes pri-miRNAs generated in their native chromatin context and how nuclear pri-miRNA processing is linked to other gene processes remain, however, poorly documented (Pawlicki and Steitz, 2010). In plants, pri-miRNA transcripts, DCL1 and HYL1 (functionally analogous to Drosha and DGCR8, respectively) concentrate in Cajal body-like structures, the so-called nuclear dicing bodies, suggesting that miRNA biogenesis and/or storage of miRNA processing factors are compartmentalized (Fang and Spector, 2007; Fujioka et al., 2007; Song et al., 2007). In animals, although a role has been suggested for the nucleolus (Politz et al., 2009; Politz et al., 2006; Shiohama et al., 2007; Wu et al., 2000), no intranuclear processing sites have been documented so far. Biochemical assays or the visualization of ectopically expressed artificial pri-miRNAs suggest that Microprocessor acts cotranscriptionally, before RNA splicing (Ballarino et al., 2009; Kataoka et al., 2009; Kim and Kim, 2007; Morlando et al., 2008; Pawlicki and Steitz, 2008; Pawlicki and Steitz, 2009).

To obtain a more physiological view of how miRNA biogenesis is organized within nuclear space, we designed a cell-imaging approach that allows for the first time imaging of endogenously expressed mammalian pri-miRNAs from newly synthesized transcripts generated in their natural chromatin context to released pri-miRNAs species dispersed throughout the nucleoplasm. As a model, we selected the primate- and placenta-specific chromosome 19 miRNA cluster (C19MC) that harbors 46 highly related pre-miRNAs processed from the repeated introns of the RNA Pol-II-transcribed non-proteincoding C19MC-HG (host-gene) pri-miRNA transcripts (Bentwich et al., 2005; Bortolin-Cavaillé et al., 2009). We report that $\mathrm{C} 19 \mathrm{MC}$ has the ability to recruit a large fraction of Microprocessor that binds onto newly released, unspliced primiRNAs retained in close proximity to their transcription sites rather than to truly nascent pri-miRNAs still attached to their genes. We suggest a model whereby DGCR 8 and Drosha are targeted to C19MC pri-miRNA as a pre-formed complex from which they independently dissociate.

\section{Results}

Distribution of C19MC-HG pri-miRNA transcripts from their transcription sites to the nucleoplasm

To visualize newly synthesized C19MC-HG pri-miRNA transcripts, we took advantage of their repeated nature to develop high-resolution RNA fluorescence in situ hybridization (FISH) methodologies using short DNA oligonucleotide probes designed to hybridize within repeated sequences of C19MC primiRNAs (Fig. 1A). As shown in (Fig. $1 \mathrm{Ba}-\mathrm{c})$, two different oligo-probes, matching either upstream or downstream of the intronic pre-miRNA sequence, revealed a single, relatively large nuclear RNA signal per nucleus in most, if not all, of the choriocarcinoma JEG3 cells used as a model. These intronic signals correspond to unspliced pri-miRNA transcripts, and not to spliced-out introns, because they colocalize perfectly with exonic signals (Fig. 1Bd-f), as well as with signals from probes spanning unspliced exon-intron junctions (Fig. 1Bg-i). Although C19MC-HG is spliced in JEG3 cells, RNA FISH failed to detect fully spliced nucleoplasmic pri-miRNAs (not shown), indicating that the latter might represent labile RNA species. Surprisingly, $40-50 \%$ of these pri-miRNA FISH signals have characteristic doublet or dumbbell-like structures (Fig. 1B, enlarged insert) in which the bulk of the two RNA signals is positioned $\sim 1.6 \mu \mathrm{m}$ apart $(n=102)$, whereas in some nuclei (5$10 \%$ ) even more complex RNA shapes occupying a significant part of the nucleoplasm can also be observed (Fig. 1C; see also (Noguer-Dance et al., 2010). The significance of such structurally constrained RNA species that appear to be impeded in their ability to diffuse freely remains elusive. They are not artifacts due to our hybridization procedure because they are also seen in living cells (supplementary material Movie 1).

Given their proximity to DNA FISH signals (Fig. 1Da-c; supplementary material Fig. S1) and because they can extend well beyond them (Fig. 1D, bottom), these pri-miRNA FISH signals most probably correspond to released transcripts retained near C19MC transcription sites. In more than $80 \%$ of nuclei, C19MC pri-miRNA FISH signals are preferentially, if not exclusively, expressed from only one allele; an observation in agreement with the imprinting of C19MC (Noguer-Dance et al., 2010). DNA FISH signals were frequently found as doublets, or even more complex pinpoint DNA FISH signals (Fig. 1D, bottom) that presumably reflect specific higher-order chromatin organization at highly expressed, tandemly repeated arrays of non-coding DNA in decondensed chromatin. Indeed, they recall DNA FISH patterns recently documented at three other imprinted repeated small RNA gene clusters that, notably, also give rise to huge amounts of nuclear-retained repeated non-coding RNA species (Leung et al., 2009; Vitali et al., 2010). Whether high expression of imprinted non-coding RNA genes plays a role in chromatin structure is an appealing hypothesis that needs to be tested experimentally.

Numerous punctate and weaker RNA dot-like signals (stained by both intronic and exonic probes) were also detected (Fig. 1E, enlarged inserts). Given that $\sim 60 \%$ of these dots were positioned relatively close to the bulk of RNA signals (supplementary material Fig. S2A), we hypothesized that they might represent single molecules of unspliced pri-miRNAs that emanate from C19MC transcription sites and diffuse throughout the nucleoplasm. We quantified the number of hybridized intronic probes within each dot, reasoning that a number of probes largely exceeding $\sim 46$ (the largest theoretical number of probes per molecule for full length pri-miRNA, i.e. C19MC hosts 46 miRNA genes embedded within repeated units; Fig. 1A) would not correspond to the single RNA molecule hypothesis (Femino et al., 1998). We found that $62.1 \%(625 / 1006)$ contained a limited number of intronic probes ranging from 4 to 13 (Fig. 1F), strengthening the notion that RNA dots might represent single (or perhaps aggregates of a very few) RNA molecule(s). Their fluorescence intensities correlated with their distribution relative to the bulk of RNAs, the closest being the brightest (supplementary material Fig. S2B), suggesting that those nucleoplasmic pri-miRNAs undergo Microprocessor-mediated cleavages and/or RNA decay, with truncated pri-miRNA molecules containing less complementarities to oligo-probes.

\section{Bulk recruitment of the Drosha-DGCR8 complex occurs onto retained pri-miRNA species in the vicinity of C19MC genes}

We asked next whether Microprocessor could be visualized on newly synthesized pri-miRNAs at the C19MC locus. RNA FISH experiments were followed by the detection of endogenous Drosha 
A

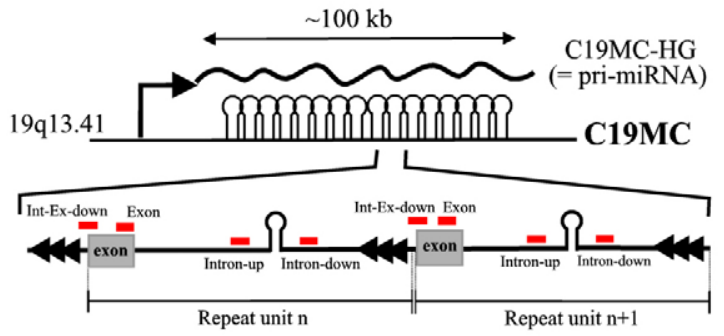

C

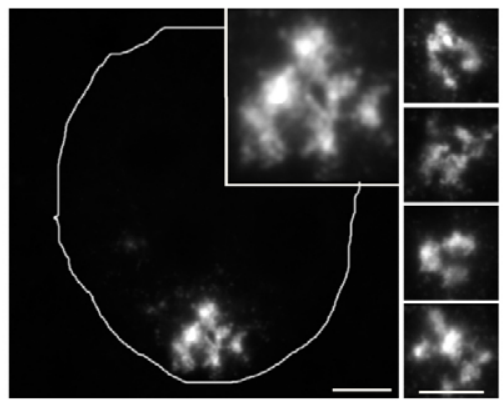

B
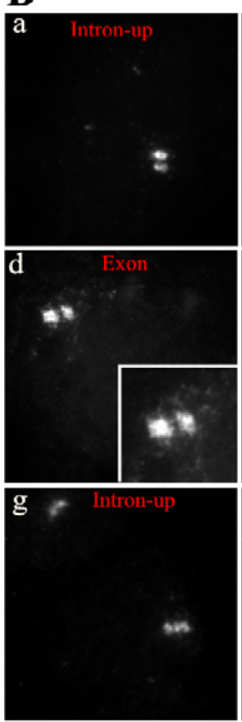

E
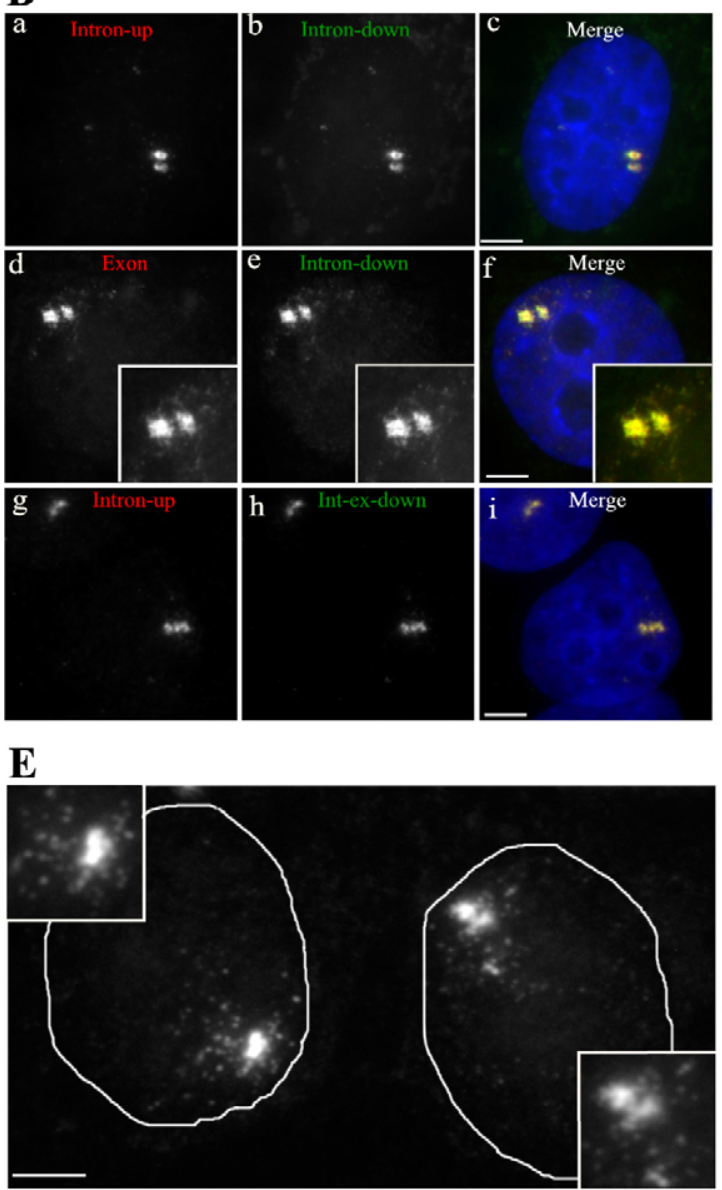

D
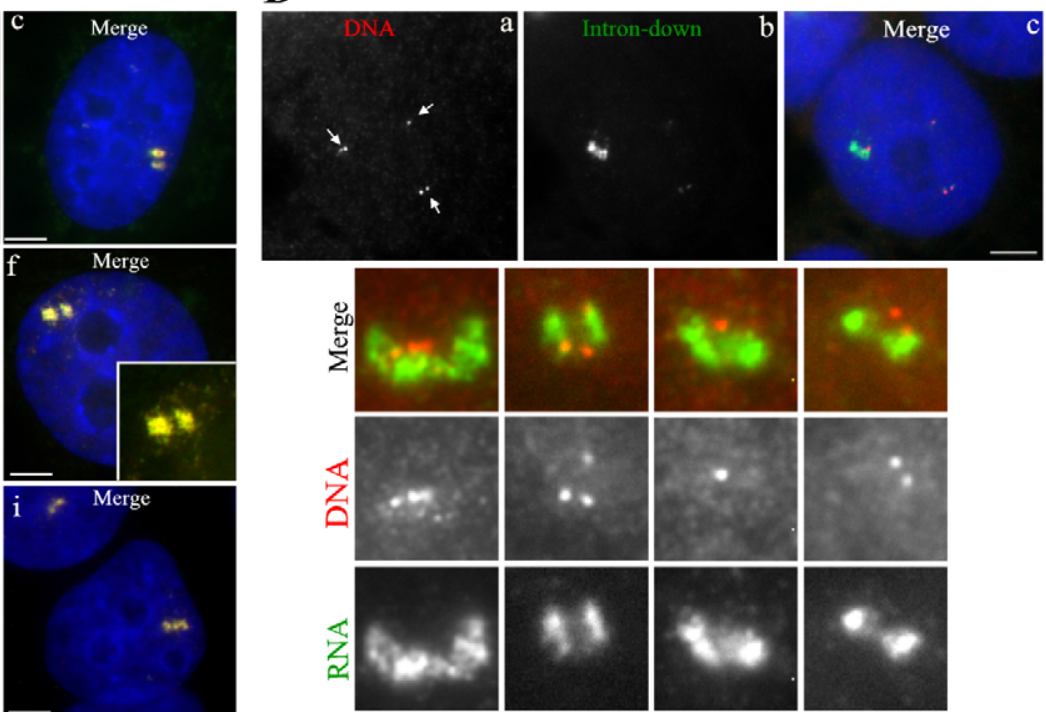

F

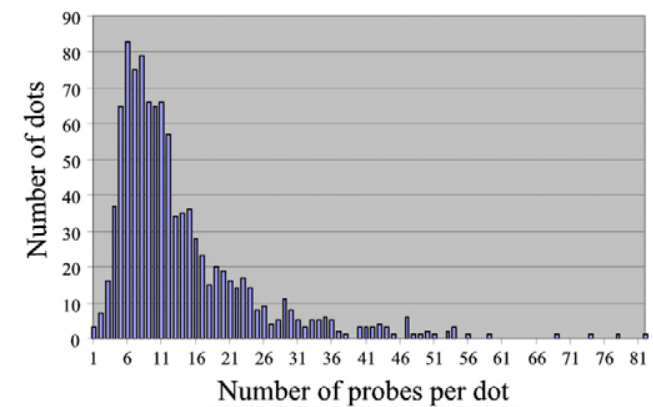

Fig. 1. Intranuclear distribution of C19MC pri-miRNA transcripts at the single-nucleus level. (A) Schematic representation of the array of repeated C19MC miRNA genes ( $\sim 100 \mathrm{~kb}$ in length) at the human Chr19q13.41 locus (Bortolin-Cavaillé et al., 2009). Most of the 46 highly related pre-miRNAs (symbolized as stem-loops) are embedded within repeated introns of long non-protein-coding RNA (termed C19MC-HG), denoted by the wavy line. Repeated exons of C19MCHG and flanking antisense-oriented Alu elements are represented as gray boxes and black triangles, respectively. The relative positions of oligo-probes used for RNA FISH are indicated by the red bars (two repeated units are shown). (B) Intranuclear distribution of unspliced pri-miRNA transcripts in JEG3 cells hybridized with Cy3-labeled intron-up (red) and Alexa-Fluor-488-labeled intron-down (green) probes (Ba-Bc), with Cy3-labeled exonic (red) and Alexa-Fluor-488-labeled intron-down (green) probes (Bd-Bf), or with Cy3-labeled intron-up (red) and Cy5-labeled intron-exon junction (green) probes (Bg-Bi). (C) Examples of large, structurally constrained RNA signals revealed by Cy3-labeled intron-down probes. The white line delineates nuclear boundaries as judged by DAPI staining. (D) DNA/RNA FISH (Da-Dc) was carried out with a mixture of four Cy3-labeled probes designed to recognize the template strand of C19MC genes (DNA, red) and with Alexa-Fluor-488-labeled intron-down probes (RNA, green). White arrows indicate the position of the three DNA FISH signals: most JEG3 cells contain three chromosome 19 and three C19MC loci, as revealed by DNA FISH performed on metaphase chromosomes (supplementary material Fig. S1). Bottom panels show the spatial organization of newly made C19MC pri-miRNA transcripts relative to their genes. Unspliced pri-miRNAs (green signals) extend largely beyond C19MC genes (red signals). (E) Two nuclei displaying nucleoplasmic dot-like RNA signals around C19MC transcription sites, revealed by a Cy3-labeled intronup probe (enlarged insets). The contrast has been enhanced to highlight pri-miRNA dots. (F) Histograms show the total fluorescence intensity of each dot (1006 individual nuclear dots in 26 different nuclei) converted into the number of hybridized probes. Scale bars: $5 \mu \mathrm{m}$. 
A

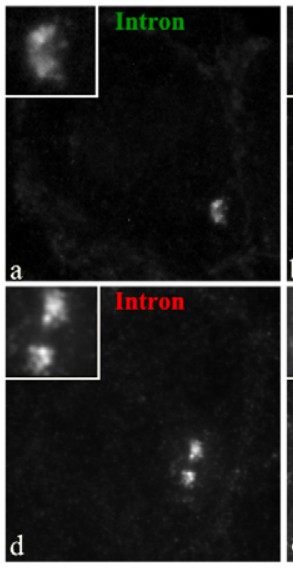

C

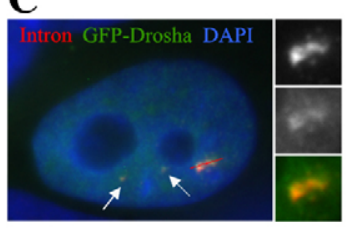

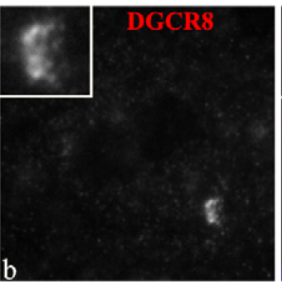
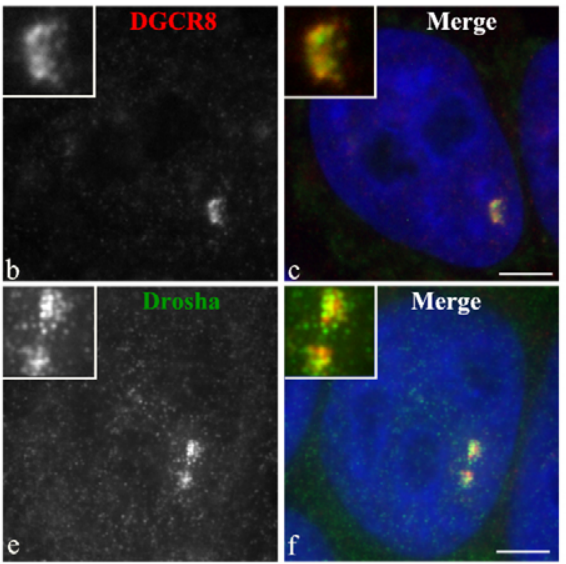

B

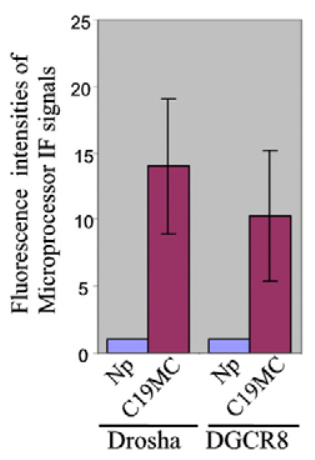

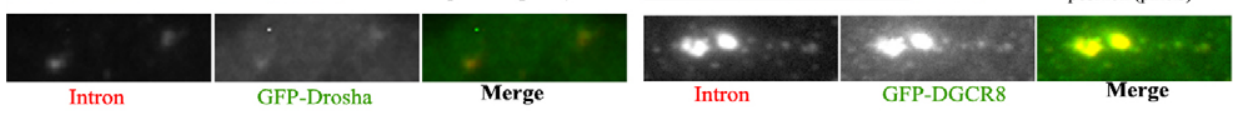
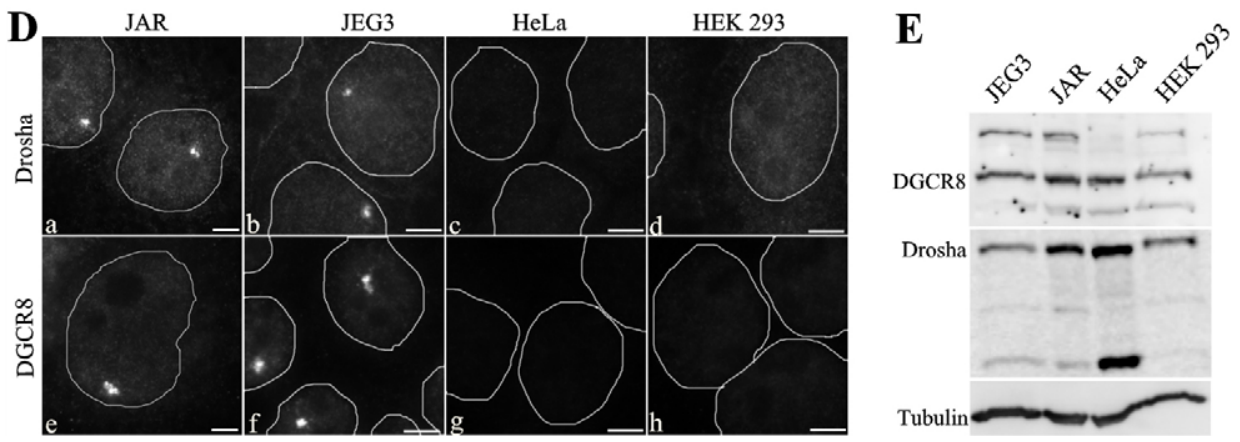

Fig. 2. Microprocessor is recruited massively to the C19MC locus. (A) Partial colocalization of DGCR8 (Aa-Ac) and Drosha (Ad-Af) with unspliced primiRNAs at C19MC. JEG3 cells were hybridized with Alexa-Fluor-488- (green) or Cy3-labeled (red) intron probes followed by immunostaining of endogenous DGCR8 (red) and Drosha (green), respectively. (B) Histograms show the 2D fluorescence intensities of Drosha and DGCR8 immunofluorescence signals at C19MC (red histograms) or at other randomly chosen nuclear regions (Np) excluding nucleoli (blue histograms). Values represent the mean \pm s.d. (75 and 109 nuclei were analyzed for Drosha and DGCR8, respectively). The Np fluorescence intensity was set to 1. (C) GFP-Drosha (left, green) and GFP-DGCR8 (right, green) are faithfully targeted to C19MC transcription sites visualized by Cy3-labeled intron probes (red). The plot to the right shows fluorescence intensities of unspliced pri-miRNA (red) and GFP-Microprocessor (green) signals across the red line. White arrows indicate 'silent alleles' that display transcription leakage. Bottom left panel: enlarged inserts showing that recruitment of GFP-Drosha can be seen at weakly expressed silent alleles. Bottom right panel: enlarged inserts from another nucleus showing some RNA dot-like signals that colocalize with GFP-DGCR8. (D) Clustering of DGCR8 and Drosha at C19MC is observed in C19MC-expressing cells (Da,Db,De,Df), but not in C19MC non-expressing cells (Dc,Dd,Dg,Dh). HeLa and HEK293 images were processed in the same manner as JEG3 images to allow direct comparison (supplementary material Fig. S4). (E) Western blots on cellular lysates with anti-DGCR8, anti-Drosha and anti-tubulin (gel-loading control) antibodies. The identity of the two additional bands seen for DGCR8 and Drosha, whose relative intensities can vary from one experiment to other, is unknown. Scale bars: $5 \mu \mathrm{m}$.

or DGCR8 proteins by indirect immunofluorescence. As shown in Fig. 2A, strong Drosha and DGCR8 immunofluorescence signals overlap partially with those of unspliced pri-miRNAs at C19MC. With these image time-acquisition settings, only a relatively weak Microprocessor immunofluorescence signal could be detected in the nucleoplasm, notably for DGCR8. Punctate immunofluorescence Drosha signals were frequently observed throughout the nucleoplasm, but also in the cytoplasm. Their specificity is, however, questionable because they persist to some extent in Drosha-depleted cells (Fig. 4) and their relative intensities can vary greatly from one experiment to another. Importantly, two-dimensional quantitative fluorescence analysis showed that C19MC contains $\sim$ ten- to 15 -fold more Microprocessor immunofluorescence signal than any other randomly chosen nucleoplasmic region (Fig. 2B).

Microprocessor recruitment at $\mathrm{C} 19 \mathrm{MC}$ requires ongoing transcription because Microprocessor signals disappeared in actinomycin D (AMD)-treated cells (not shown) and 
A
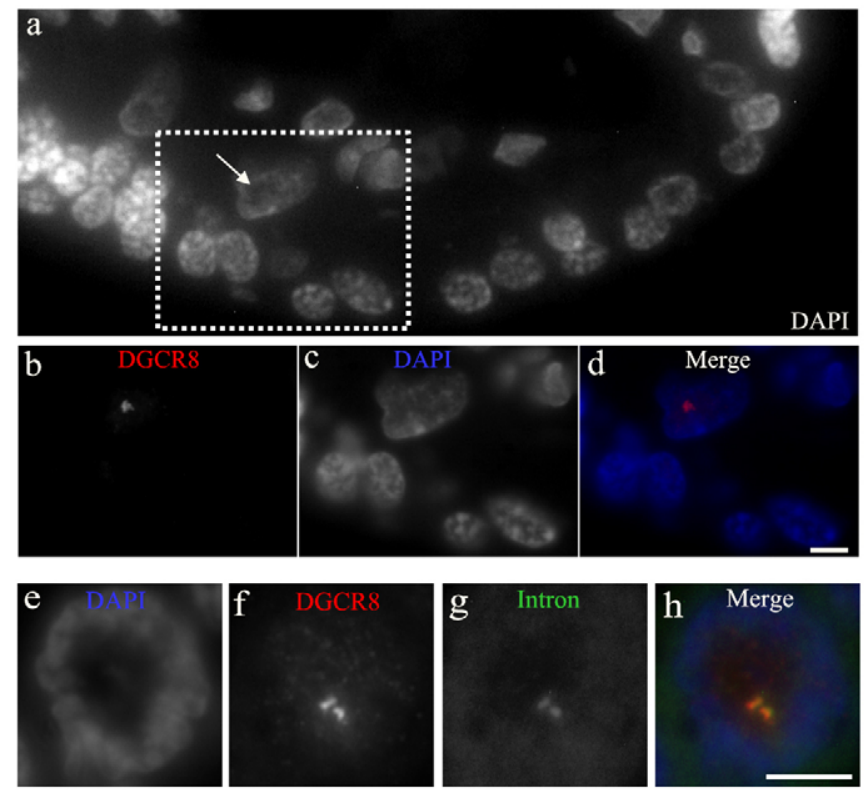

B

$\mathrm{HeLa}+5$-aza ( $2 \mu \mathrm{M} / 3$ days $)$

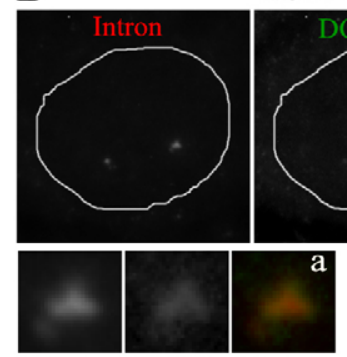

Intron DGCR8 Merge

Fig. 3. Endogenous DGCR8 localizes to C19MC in C19MC-expressing non-choriocarcinoma cells. (A) Immunofluorescence alone (Aa-Ad) or FISH coupled to immunofluorescence (Ae-Ah) was performed on 3- $\mu \mathrm{m}$ paraffin-embedded placenta sections with Cy5-labeled intronic probes (green) and anti-DGCR8 antibodies (red). The white box (dotted lines) indicates the area enlarged in $\mathrm{Ab}-\mathrm{Ad}$ and the white arrow points to a DGCR8-positive cell. Doublet-like pri-miRNA FISH signals observed in another cell are shown in Ae-Ah. (B) DGCR8 localizes to epigenetically de-repressed C19MC alleles in 5-aza-treated HeLa cells. The white line shows the DAPI-delineated nuclear boundary. Bottom: enlarged inserts showing colocalization of DGCR8 at two expressing C19MC alleles $(\mathrm{a}, \mathrm{b})$ indicated by white arrows. Right: examples of doublet-like FISH signals seen at two other epigenetically de-repressed C19MC alleles. Scale bars: $5 \mu \mathrm{m}$.

recruitment occurs on pri-miRNA transcripts that mostly occupy chromatin-poor nuclear domains that do not correspond to regions of active transcription, without any preferential colocalization with known nuclear bodies or RNA Pol-IIenriched regions (supplementary material Fig. S3). Given that RNA signals can extend far beyond DNA signals (Fig. 1D), these observations support the idea that most pri-miRNAs are detached from the chromatin and, as a corollary, that Microprocessor functions mostly at the post-transcriptional level in the vicinity of C19MC genes.

Transiently expressed GFP-tagged DGCR8 or Drosha proteins (Fig. 2C) also localize to the bulk of pri-miRNAs at C19MC and can be detected at some imprinted alleles where very weak transcription leakage occurs, as judged by the faint intensities of their associated FISH signals (Fig. 2C, left and enlarged panels). In addition, some, but not all, of the pri-miRNA dot-like signals described above associate with GFP-DGCR8 (16\%) but apparently not, or very rarely, with GFP-Drosha signals (3\%) (Fig. 2C, right and enlarged panels). Because these GFP dots are also preferentially found in close proximity to C19MC genes (supplementary material Fig. S2A), they are likely to represent DGCR8-bound pri-miRNAs leaving C19MC transcription sites (supplementary material Movie 1). Unfortunately, the relatively low signal-to-background ratio has so far limited any further characterization of the motion of these individual pri-miRNPs in the nucleus of living cells.

Accumulation of endogenously expressed Microprocessor at C19MC was also seen in two other choriocarcinoma cell lines expressing C19MC, JAR (Fig. 2D) and BeWo (not shown). By contrast, this was not the case in HeLa, HEK 293, T24 or U2OS cells that did not express C19MC miRNA genes and which instead displayed diffuse Microprocessor nucleoplasmic signals (Fig. 2D; see also supplementary material Fig. S4). Given that the expression levels of Drosha (Fig. 2E) and DGCR8 were of comparable magnitude in these lines, we conclude that a significant amount of Microprocessor accumulates and functions at active $\mathrm{C} 19 \mathrm{MC}$ miRNA genes.

Why does C19MC have the apparent ability to attract Microprocessor, and is this phenomenon restricted to choriocarcinoma cells? To address these important questions, we first assessed the presence of Microprocessor at C19MC in vivo by examining the intranuclear distribution of DGCR8 immunofluorescence and pri-miRNA FISH signals in paraffinembedded sections of human placenta collected at term. C19MC pri-miRNA FISH signals were detected in the nuclei of some, but not all, trophoblasts $(\sim 5 \%)$, which, based on the appearance of their DAPI staining and their relative inner position along the villi, very probably represent cytotrophoblasts (Fig. 3A). Interestingly, DGCR8 immunofluorescence signals were found at C19MC transcription sites in the vast majority of these nuclei, indicating that massive recruitment of Microprocessor at C19MC also occurs in physiologically relevant tissues. We also treated C19MC non-expressing HeLa cells with the DNA methylation inhibitor 5-aza-2'-deoxycytidine (5-aza), which can partially reactivate $\mathrm{C} 19 \mathrm{MC}$ expression in these cells (Noguer-Dance et al., 2010). As illustrated in Fig. 3B, endogenous DGCR8 was localized to these epigenetically de-repressed C19MC alleles, demonstrating that $\mathrm{C} 19 \mathrm{MC}$ retains the intrinsic ability to recruit Microprocessor in cells that are not of placental origin. Relatively modest amounts of pri-miRNA species are usually generated in placenta or 5-aza-treated HeLa cells compared with choriocarcinoma cells, yet substantial numbers of FISH signals adopted the characteristic doublet-like structures discussed above. This indicates that these structurally constrained RNA FISH signals reflect a distinct property of the C19MC locus that is very probably independent of the local deposition of large amounts of RNAs.

\section{DGCR8 is essential for stable recruitment of Drosha onto C19MC pri-miRNA transcripts}

Drosha and DGCR8 were knocked down by RNA interference (RNAi) to evaluate the impact of DGCR8 depletion on the intranuclear distribution of Drosha and vice versa. Only 20\% of JEG3 cells transiently transfected with small interfering RNAs 
A

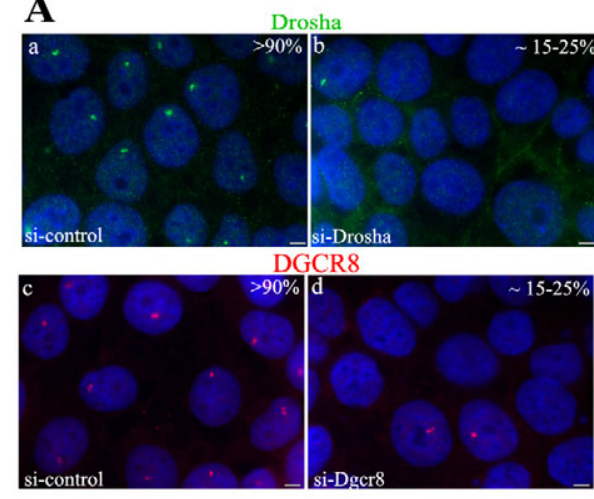

B
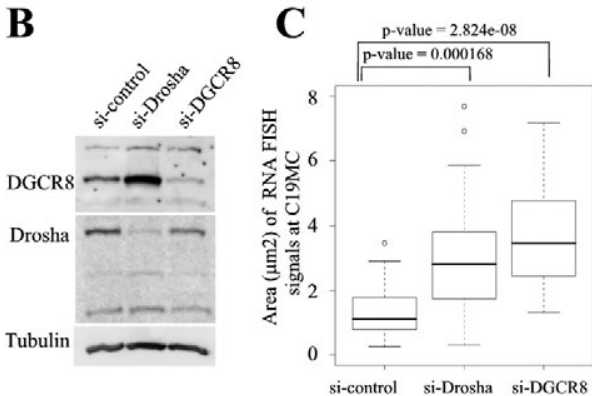

$\mathbf{F}$
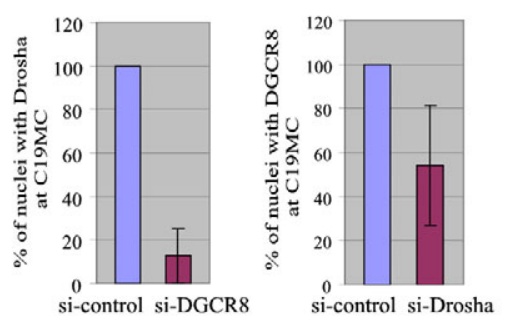

D
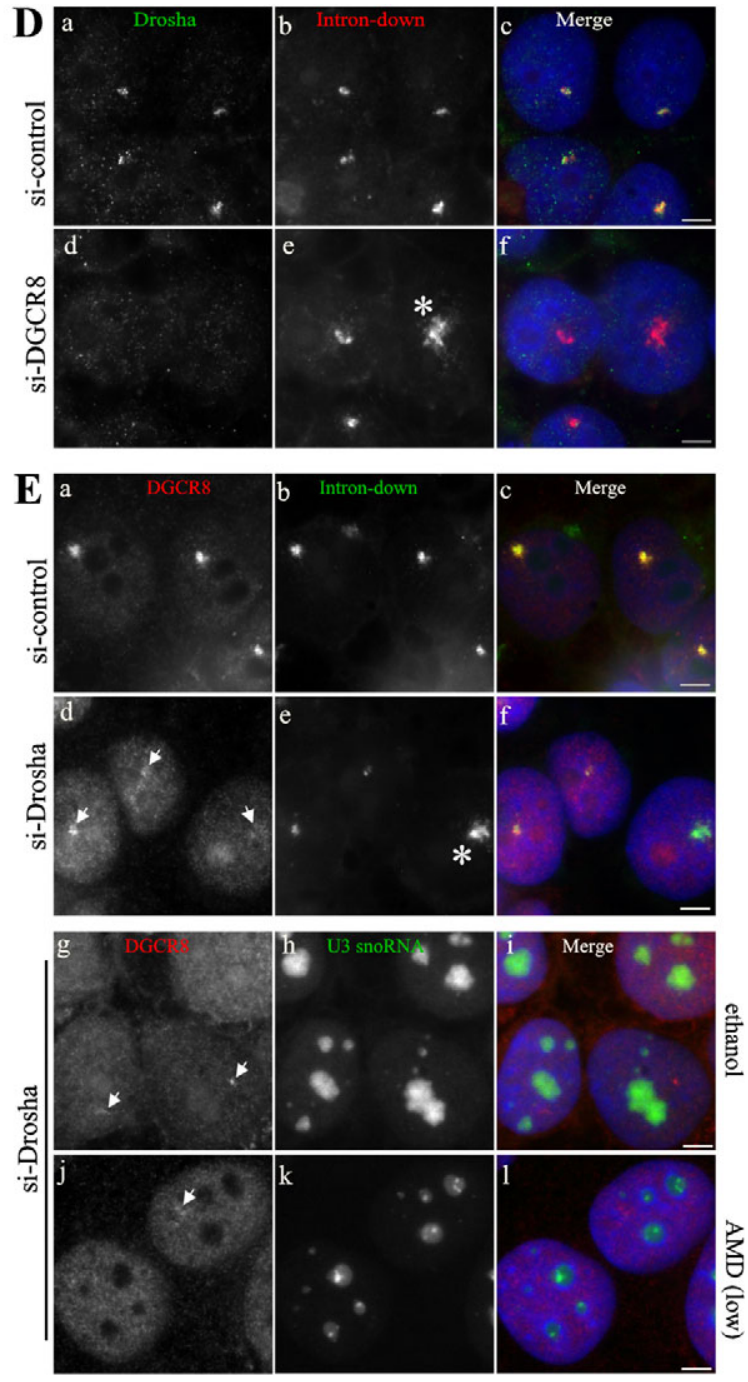

Fig. 4. Recruitment of Drosha and DGCR8 onto C19MC-HG transcripts is interdependent. (A) RNAi knockdown of the Microprocessor (Drosha-DGCR8) complex. Representative field of JEG3 cells transiently transfected with control siRNA (Aa,Ac) or with siRNAs targeting Drosha (Ab) or DGCR8 (Ad) and then immunostained with antibodies against Drosha (green; Aa,Ab) or DGCR8 (red; Ac,Ad). The percentage of nuclei with clear nuclear signals at C19MC is indicated. At least 200-300 nuclei were scored. (B) Western blots of JEG3 lysates transfected with siRNAs against Drosha or DGCR8 and probed with the antibodies indicated on the left. (C) Box plots represent the areas of unspliced pri-miRNA FISH signals at C19MC in control or in Microprocessor-depleted cells. 30 nuclei were scored and Wilcoxon test $P$ values are given. (D) The colocalization of Drosha with unspliced C19MC pri-miRNA is impaired in DGCR8-depleted cells. JEG3 cells transfected with control siRNA (Da-Dc) or with siRNA targeting DGCR8 (Dd-Df) were hybridized with Cy3-labeled (red) intronic probes followed by immunostaining for Drosha (green). (E) DGCR8 relocalizes in the nucleoplasm and in the nucleolus in Drosha-silenced cells (Ea-Ef). JEG3 transfected with control siRNA (Ea-Ec) or siRNA targeting Drosha (Ed-Ef) were hybridized with Alexa-Fluor-488-labeled (green) intronic probes followed by immunostaining for DGCR8 (red). The nucleolar redistribution of DGCR8 requires Pol I transcription (Eg-El). Drosha-silenced cells were treated with ethanol (Eg-Ei) or actinomycin D (AMD; $0.04 \mu \mathrm{g} / \mathrm{ml}$ for 60 minutes; Ej-El) and hybridized with Cy5-labeled U3 snoRNA probes used as a nucleolar marker (green) followed by immunostaining for DGCR8 (red). Small arrows indicate DGCR8 immunofluorescence signals whose detection is hampered to some extent by the increase in nucleoplasmic signals. Asterisks in D and E indicate representative large RNA FISH signals revealed in DGCR8- and Drosha-depleted cells, respectively. (F) Histograms show the percentage of nuclei with Drosha at C19MC in DGCR8-silenced (left) or with DGCR8 at C19MC in Drosha-depleted cells (right). The percentage of Microprocessor signals was set at $100 \%$ in cells transfected with control siRNAs. Values represent the mean \pm s.d. of three independent RNAi experiments ( $\sim 200-400$ nuclei were scored for each experiment). Scale bars: $5 \mu \mathrm{m}$.

(siRNAs) directed against mRNAs encoding DGCR8 or Drosha exhibited detectable immunofluorescence signals of the targeted proteins at $\mathrm{C} 19 \mathrm{MC}$ (Fig. 4A). In agreement with the reported cross-regulation between DGCR8 and Drosha (Han et al., 2009), depletion of Drosha led to an increase in DGCR8 levels (Fig. 4B). However, in our system, the nuclear content of Drosha remained unaffected in DGCR8-silenced cells. Note that, in Microprocessor-depleted cells, the steady-state level of C19MC pri-miRNA transcripts is upregulated (BortolinCavaillé et al., 2009). This, importantly, is accompanied by a significant increase in the proportion of nuclei with large primiRNA FISH signals (Fig. 4C), indicating that Microprocessor not only binds in proximity to transcription sites but most likely also cleaves there. In agreement with the idea that RNA dots 
represent released DGCR8-bound pri-miRNAs, their brightness and their number per nucleus increased in DGCR8-silenced cells (supplementary material Fig. S2).

The proportion of nuclei with Drosha immunofluorescence signals at C19MC was dramatically reduced in DGCR8-depleted cells (Fig. 4Dd, 4F), supporting the notion that DGCR8 plays a role in recruiting Drosha and/or stabilizing its binding to the primiRNAs (Han et al., 2006; Yeom et al., 2006). Remarkably, significant DGCR8 signals were still detected in a substantial number of nuclei silenced for Drosha, even though quantification showed an apparent decrease in DGCR8 recruitment (Fig. 4Ed, 4F). More surprising is the observation that, in the absence of Drosha, DGCR8 redistributed throughout the nucleus, including the nucleolus. Because DGCR8 is excluded from the nucleolus in cells treated with a low concentration of AMD $(0.04 \mu \mathrm{g} / \mathrm{ml})$, which specifically inhibits RNA polymerase I (Fig. 4Ej), we assume that excess free nucleoplasmic DGCR8 is sequestered in the nucleolus through nonspecific interactions with ribosomal RNAs. Intriguingly, DGCR8, but also some miRNAs, have been previously found in the nucleolus of non-placental-derived cells (Politz et al., 2006; Politz et al., 2009; Shiohama et al., 2007). Whether DGCR8 traffics rapidly within the nucleolus of JEG3 cells under normal conditions remains an open question. Taken together, these results indicate that the intranuclear distributions of DGCR8 and Drosha are interdependent, but that depletion of DGCR8 is much more detrimental to Drosha localization at C19MC than the converse.

\section{GFP-DGCR8 can be targeted to C19MC in a Drosha- independent manner}

To delineate the role of protein domains important for targeting to $\mathrm{C} 19 \mathrm{MC}$, we assayed the intranuclear fate of transiently transfected GFP-tagged Drosha and DGCR8 mutants whose biochemical proprieties have been extensively characterized in vitro (Han et al., 2004; Lee et al., 2006; Yeom et al., 2006). As shown in Fig. 5A (see also supplementary material Fig. S5A), Nterminal deletions of the proline-rich domain of Drosha (Drosha $\Delta N 220$, Drosha $\Delta N 135$ and Drosha $\Delta$ N65) that keep intact its association with DGCR8 in vitro did not completely impair recruitment to $\mathrm{C} 19 \mathrm{MC}$, even though GFP signals at C19MC for these mutants, particularly those of GFPDrosha $\Delta N 220$, were lower than those of full-length GFPDrosha. The same holds true for GFP-Drosha $\Delta \mathrm{C} 114$ and Drosha $\Delta C 432$ mutants, whose signals at C19MC could still be detected in some, but not all nuclei. Although signals were relatively weak, or even barely detectable, these results nevertheless show that the Drosha double-stranded RNAbinding domain (dsRBD) is not absolutely required for primiRNA binding. Finally, Drosha $\Delta$ N390 and Drosha $\Delta$ N490 mutants mostly localized in the cytoplasm and were never seen at C19MC.

By contrast, deleting the C-terminal domain of DGCR8 that contains the two double-stranded RNA-binding domains (DRBD) [DGCR8(1-483)] or mutating the DRBDs strongly affected recruitment to C19MC, whereas DGCR8(1-692) mutants lacking the C-terminal Drosha-binding site (Yeom et al., 2006) still precisely localized to $\mathrm{C} 19 \mathrm{MC}$ (Fig. 5B; see also supplementary material Fig. S5B). GFP-DGCR8 $\Delta \mathrm{WW}$ mutants (deleted for the WW domain) were also dramatically affected, suggesting that interaction with an additional factor might facilitate DGCR8 binding to $\mathrm{C} 19 \mathrm{MC}$ transcripts. Although ectopically expressed
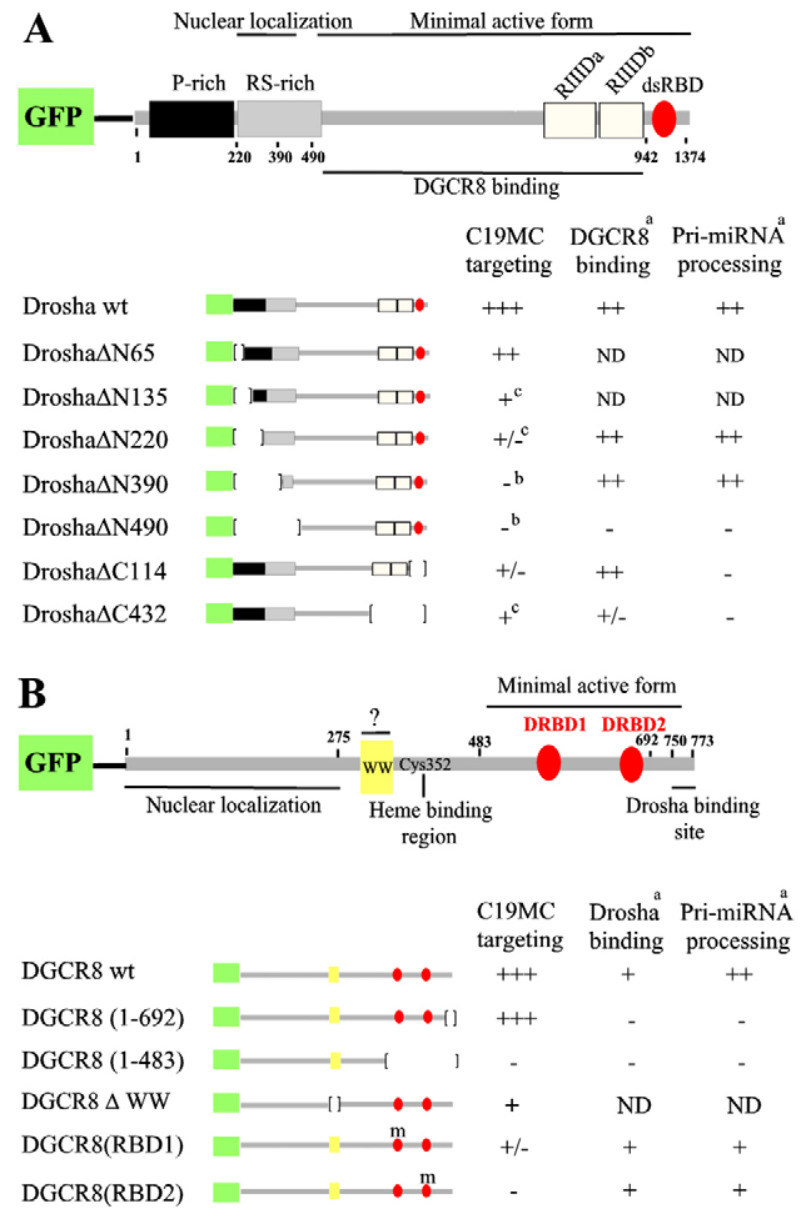

Fig. 5. Identifying the protein domains required for Microprocessor targeting to the C19MC locus. (A) Summary of the intra-nuclear distribution of GFP-Drosha mutants. (B) Summary of the intra-nuclear distribution of GFP-DGCR8 mutants. The domain organizations of Drosha and DGCR8, as reviewed (Lee et al., 2006), are shown: P-rich, proline rich domain; RS-rich, arginine- and serine-rich domain; RIIID, RNase III domain; dsRBD, doublestranded RNA-binding domain; DRBD, double-stranded RNA-binding domain. Some transfected nuclei are shown in supplementary material Fig. S5. +++ , clear GFP signals detected by eye in most examined nuclei; ++ , clear GFP signals in most, but not all nuclei; + , weak GFP signals in most nuclei; +/-, weak GFP signals in a subset of nuclei; - , no GFP signals; ND, not determined; superscript a, based on published studies (Faller et al., 2007; Han et al., 2004; Han et al., 2006; Yeom et al., 2006); superscript b, GFPtagged proteins mostly localize within the cytoplasm; superscript c, GFPtagged proteins display a nuclear speckle-like pattern in a subset of nuclei; m, mutated.

GFP mutants could possibly follow different intranuclear paths relative to endogenously expressed Microprocessor, this mutagenesis analysis extends the conclusions drawn from our RNAi experiments: DGCR8 can be targeted to C19MC in a Drosha-independent manner.

\section{GFP-DGCR8 and GFP-Drosha display slow release kinetics near C19MC genes}

An important issue is whether Microprocessor at the C19MC locus is in continuous flux with other nuclear regions, i.e. does it rapidly dissociate from, and re-associate with, the C19MC locus? To address the dynamics of Microprocessor at C19MC, JEG3 
cells transiently transfected with either GFP-DGCR8 or GFPDrosha were subjected to fluorescence recovery after photobleaching (FRAP). As shown in Fig. 6A-C, fluorescence of GFP-DGCR8 at C19MC recovered to $79 \%$ of pre-bleach intensity within 34 minutes, with a recovery $t_{1 / 2}$ of $\sim 7$ minutes. By contrast, when GFP-DGCR8 signals were bleached randomly in the nucleoplasm, the fluorescence recovered very quickly, with a $t_{1 / 2}$ of 3.7 seconds. Interestingly, the GFP-DGCR8(1-692) mutant that cannot bind Drosha (Yeom et al., 2006) displayed a similar $t_{1 / 2}$ of $\sim 6$ minutes (Fig. 6D). The dynamics of GFPDrosha at C19MC were also found to be slow, with a $t_{1 / 2}$ of $\sim 3$ minutes, whereas the nucleoplasmic GFP-Drosha pool displayed a $t_{1 / 2}$ of 5.65 seconds (Fig. 6B,C). Line-scan analyses of the FRAP data at C19MC transcription sites for GFP-DGCR8 and GFP-Drosha indicate that fluorescence recovery is a diffusion-uncoupled process (not shown) that reflects dissociation from pri-miRNAs in a concentration-independent
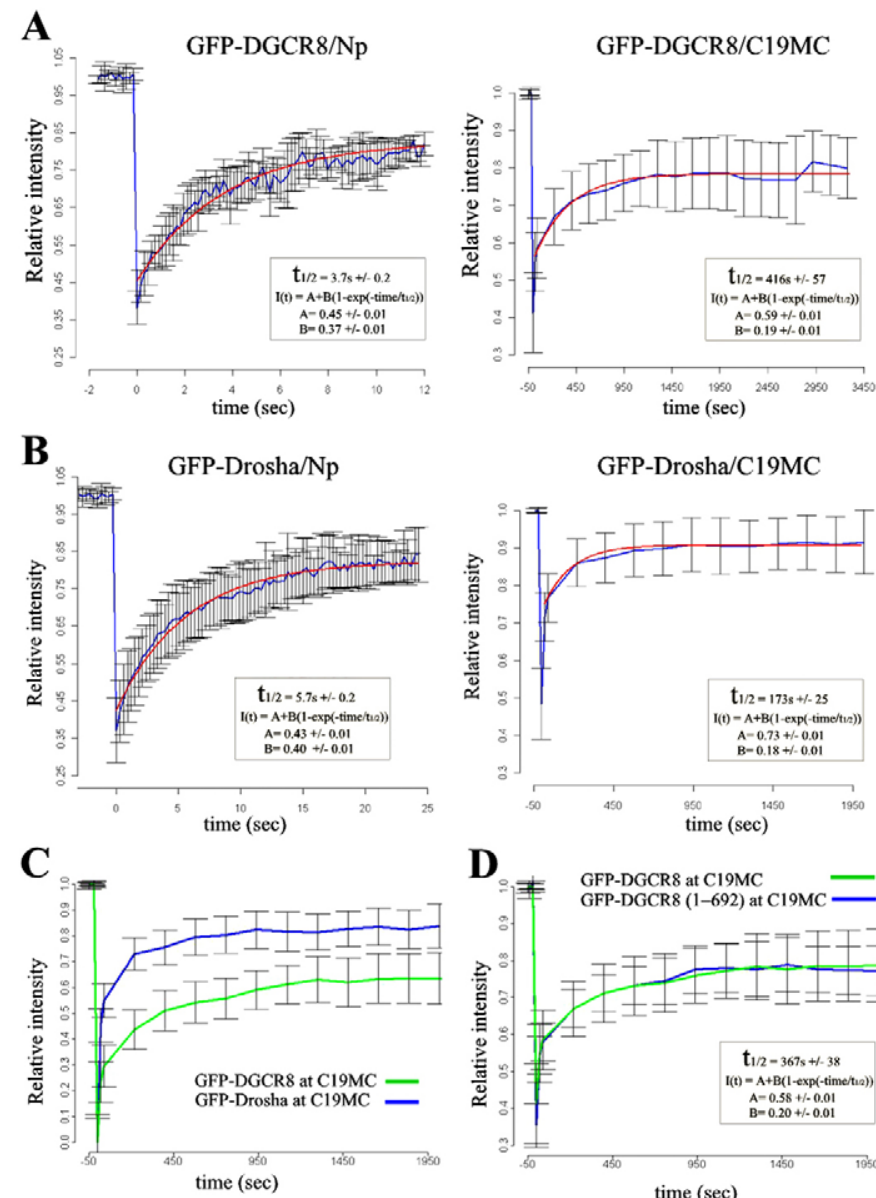

GFP-DGCR8/C19MC
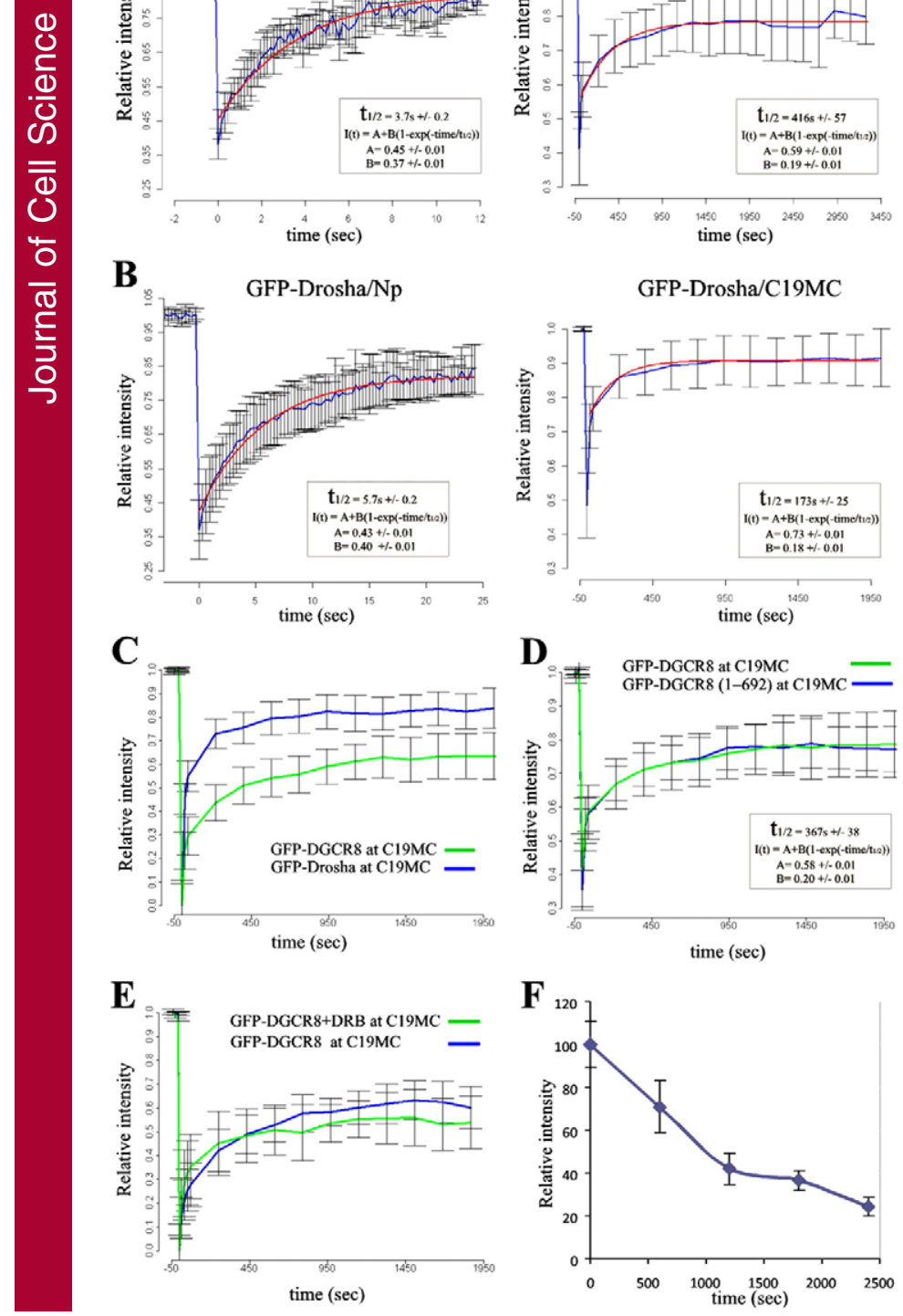

manner (Sprague et al., 2004). The immobile fraction could correspond to proteins bound to higher-affinity sites, or to proteins that directly recycle locally on the RNA, without exchanging with the nucleoplasm.

We conclude that Microprocessor at C19MC does not exchange rapidly with surrounding pools, probably due to its association with immobile nuclear binding sites that most probably correspond to pri-miRNAs constrained at $\mathrm{C} 19 \mathrm{MC}$ transcription sites. Indeed, the slow release kinetics of Microprocessor at $\mathrm{C} 19 \mathrm{MC}$, as measured for GFP-DGCR8 (Fig. 6E), remained unaltered in JEG3 cells treated with the transcription elongation inhibitor 5,6dichloro-1- $\beta$-D-ribofuranosylbenzimidazole (DRB), indicating that dissociation of Microprocessor from pri-miRNAs does not rely strictly on the activity of Pol II polymerases. Taken together, these slow exchange rates also imply that a significant fraction of Microprocessor is sequestered by the active C19MC allele, thus leaving open the possibility that Microprocessor availability might be rate-limiting for other miRNA gene loci expressed in C19MCexpressing, placenta-derived cells.

\section{Drosha and DGCR8 form a nucleoplasmic complex but dissociate separately from the $\mathrm{C19MC}$ transcription site}

The different recovery time of GFP-Drosha at C19MC relative to that of GFP-DGCR8 raises the possibility that these two proteins could be recruited onto C19MC pri-miRNA separately. Alternatively, they could be targeted as a preformed complex but leave transcription sites independently. To distinguish between these two possibilities, we applied fluorescence crosscorrelation spectroscopy (FCCS) to JEG3 cells expressing GFPDrosha and mCherry-DGCR8. The latter was found to be functional, as judged by its targeting to C19MC transcription sites (not shown). FCCS is well suited to investigation of codiffusion of GFP and mCherry fluorophores with high spatial $\left(10^{-15} 1\right.$ range) and temporal (microsecond to millisecond range) resolution (Bacia et al., 2006). Briefly, fluorescence fluctuations of these two fluorophores are measured over time, and each

Fig. 6. Microprocessor dynamics in living cells. $(\mathbf{A}, \mathbf{B})$ GFP-DGCR8 and GFP-Drosha display different exchange rates at C19MC transcription sites. The nuclei of JEG3 cells transiently transfected with GFP-DGCR8 (A) or GFP-Drosha expression plasmids (B) were photobleached for

12 milliseconds at $473 \mathrm{~nm}$, either at the site of accumulation of unspliced C19MC pri-miRNAs (right, C19MC) or at any randomly chosen area in the nucleoplasm (left, Np). Nuclei were then imaged at 10- to 180-second intervals (C19MC) or every 150-200 milliseconds (Np) and the recovery of fluorescence was monitored by time-lapse microscopy. The values are averages of results from 10-13 different nuclei for each sub-nuclear compartment. The $t_{1 / 2}$ of recovery and the values of the exponential curves that fit the data best are given in the boxes. The mono-exponential curve fit is indicated in red. (C) FRAP recovery curves of GFP-DGCR8 (green) and GFP-Drosha (blue) at C19MC are plotted together for direct comparison ( $t_{1 / 2}$ of recovery are different, $P=0.02184$, Student's $t$-test with homogeneous variances). (D) The GFP-DGCR8(1-692) mutant (blue) and wild-type GFPDGCR8 (green) display similar $t_{1 / 2}$ of recovery at C19MC $(P=0.4103)$. (E) The $t_{1 / 2}$ of recovery of GFP-DGCR8 at C19MC remains unchanged in DRB-treated cells ( $t_{1 / 2}=454 \pm 30$ seconds, $n=4$ nuclei for each condition). To take into account the decrease in fluorescence intensities at C19MC in transcriptionally arrested cells (Fig. 6F), pre-bleached intensities were corrected for each time point. (F) Intensities of GFP-DGCR8 signals at C19MC in DRB-treated cells (blue) were measured at each time point $(n=10$ nuclei). The values of fluorescence intensities after the bleach $(t=0)$ were set to 0 for $\mathrm{C}$ and $\mathrm{E}$ to allow direct comparison. Error bars indicate s.d. 


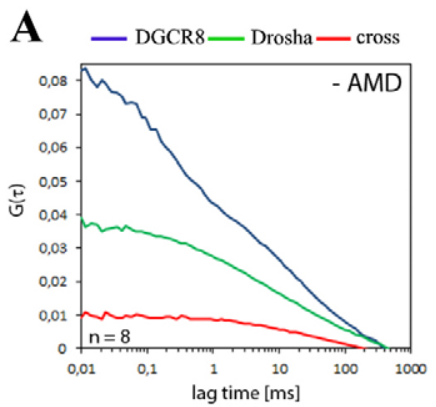

No of molecules DGCR8 20.2 +/- 14.8 Drosha $64.6+/-66.3$ $\%$ of cross correlation $16.9+/-3.6$
B

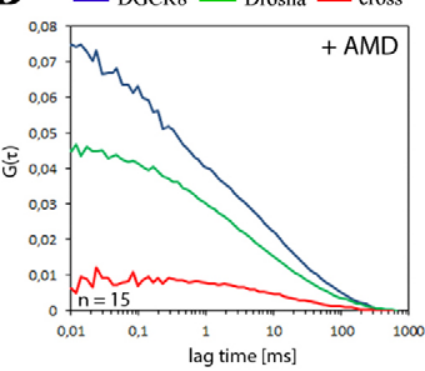

No of molecules DGCR8 27.5 +/- 28

Drosha $34.1+/-21.1$

$\%$ of cross correlation $14.9+/-10$

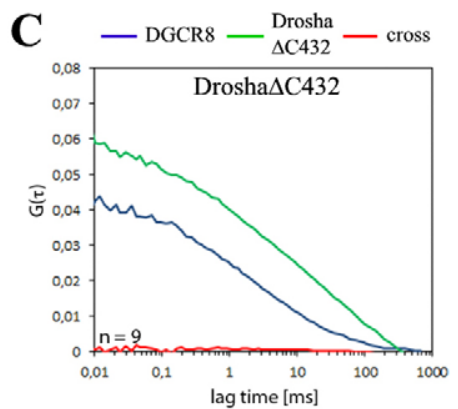

No of molecules DGCR8 21.8. +/- 33.74 Drosha $33.67+/-27.7$

$\%$ of cross correlation $1.7+/-3.6$

Fig. 7. Microprocessor in living cells. (A-C) FCCS analysis was applied to GFP-Drosha and mCherry-DGCR8. JEG3 cells were co-transfected with GFPDrosha and mCherry-DGCR8 (A), or with GFP-Drosha and mCherry-DGCR8 and pre-treated for 1 hour with $5 \mu \mathrm{g} / \mathrm{ml}$ AMD (B), or with GFP-Drosha $\Delta C 432$ and mCherry-DGCR8 (C). A laser beam was parked in the nucleoplasm and fluorescence fluctuations from both channels were collected for 120 seconds. Autocorrelation functions are plotted in green (Drosha) and blue (DGCR8), cross-correlation curves are plotted in red. The curves are averages of $n$ cells (shown in each plot).

change in fluorescence intensity means that a fluorescent molecule enters (or leaves) the observed area. Autocorrelation functions for each fluorophore can be computed from fluctuation time courses, from which concentration and diffusion properties are obtained. However, when the two labeled entities form a complex, the fluctuations of the two fluorophores are temporally synchronized. With temporally cross-correlated signals, it is then possible to compute the cross-correlation function. The degree of interaction can then be obtained by comparison with the autocorrelation functions. As shown in Fig. 7A, GFP-Drosha and mCherry-DGCR8 cross-correlate in the nucleoplasm, indicating that they interact and diffuse together.

To see whether Drosha and DGCR8 also associate in the absence of pri-miRNAs, FCCS was measured in JEG3 cells following transcriptional arrest with AMD $(5 \mu \mathrm{g} / \mathrm{ml}$ for 60 minutes), a treatment that prevents accumulation of newly synthesized pri-miRNAs at C19MC (supplementary material Fig. S6A) and also very probably at most other miRNA-encoding loci elsewhere in the nucleus. This treatment did not impair cross-correlation of Drosha and DGCR8, nor did it affect the degree of cross-correlation $(16.9 \% \pm 3.6$ and $14.9 \% \pm 10$ in control and AMD-treated cells, respectively), suggesting that DGCR8-Drosha complexes exist in the absence of transcribing Pol II (Fig. 7B). As a control, the GFP-Drosha $\Delta$ C432 mutant with impaired DGCR8 binding did not cross-correlate with mCherry-DGCR8, ruling out cross-talk between the green and red channels in our experimental setup (Fig. 7C). Hence, results of our FCCS experiments support the view that DGCR8 and Drosha reside as a complex in the nucleoplasm and that this complex can be formed in absence of transcription. Our conclusion that endogenous nuclear DGCR8 and Drosha are pre-assembled is also supported by coimmunoprecipitation assays performed in AMD-treated JEG3 cells or from RNAseA-treated cell extracts (supplementary material Fig. S6B).

\section{Discussion}

Our knowledge of how RNA synthesis, processing and trafficking are integrated within the mammalian nucleus remains relatively limited, with most live-cell imaging techniques reported so far relying on the use of artificial, tandemly repeated gene reporters (Darzacq et al., 2009). Here, we demonstrate that newly synthesized, untagged C19MC pri-miRNAs generated in their native chromatin context attract a large fraction of the DroshaDGCR8 complex (Figs 1, 2), including some Microprocessorassociated proteins and other nuclear double-stranded RNAbinding proteins (results not shown). This work provides direct visual evidence, at the gene level and in living cells, that recognition of intron-containing $\mathrm{C} 19 \mathrm{MC}$ pri-miRNAs by Microprocessor occurs in proximity to their transcription sites.

Although Pawlicki and co-workers have extensively studied the intranuclear fate of a few endogenously expressed primiRNAs in mammalian nuclei (Pawlicki and Steitz, 2008), these authors did not, to the best of our knowledge, directly visualize Microprocessor at newly synthesized pri-miRNAs. Our work therefore raises the intriguing question of why $\mathrm{C} 19 \mathrm{MC}$ attracts Microprocessor in such a remarkably efficient manner. The large amount of pri-miRNAs generated at C19MC cannot be the only explanation because Microprocessor also localizes at C19MC loci with modest, or even very faint, pri-miRNA FISH signals (Fig. 2C). There is no doubt, however, that levels of recruited Microprocessor parallel those of pri-miRNAs, implying that this striking nuclear compartmentalization is directly linked to ongoing C19MC transcription, rather than reflecting an integral part of the nuclear pri-miRNA maturation process. In contrast to ectopically overexpressed pri-miRNAs (Pawlicki and Steitz, 2008), endogenous C19MC pri-miRNAs do not accumulate in nuclear speckles (supplementary material Fig. S3). It therefore appears that expression in the native chromatin context determines the intranuclear trafficking of C19MC pri-miRNAs and also very probably their ability to attract Microprocessor. That accumulation of Microprocessor at C19MC in placenta tissues impacts on the nuclear organization and trafficking of other pri-miRNAs generated elsewhere in the genome is another appealing hypothesis that remains to be tested.

Our cell-imaging observations not only strengthen the prevailing view that pri-miRNA processing occurs shortly after 
RNA synthesis (Ballarino et al., 2009; Kataoka et al., 2009; Kim and Kim, 2007; Morlando et al., 2008; Pawlicki and Steitz, 2008) but, most importantly, also provide clues regarding targeting of Microprocessor and its mode of action at C19MC. Although the highly repetitive structure of the 100 kilobases $(\mathrm{kb}) \mathrm{C} 19 \mathrm{MC}$ locus considerably limits our understanding of transcriptional output (Bortolin-Cavaillé et al., 2009), most pri-miRNA species probably derive from a major, single transcription unit originating from promoter regions located $\sim 17 \mathrm{~kb}$ upstream of C19MC (Noguer-Dance et al., 2010). It is important to stress that our data are consistent with the view that Microprocessor acts post-transcriptionally. Indeed, given that the large pri-miRNA FISH signals lie a sizable distance away from DNA FISH signals in a Pol-II-poor region (Fig. 1; supplementary material Fig. S3) and because Microprocessor dynamics are not affected in DRBtreated cells (Fig. 6E), we favor a post-transcriptional model whereby Microprocessor binds mostly to detached RNAs deposited in close proximity to transcription sites, rather than to truly nascent pri-miRNAs still attached to their DNA template (Fig. 8). This does not imply that post-transcriptional pri-miRNA processing occurs systematically at other mammalian miRNA gene loci but suggests that it might be more prevalent than previously thought, particularly when numerous highly expressed hairpins are generated. The highly compartmentalized C19MC pri-miRNA species recall post-transcriptional 'RNA tracks' documented at several gene loci, including repeated small RNA-gene clusters (Lawrence et al., 1989; Royo et al., 2007; Shopland et al., 2002; Smith et al., 1999; Vitali et al., 2010), emphasizing the notion that RNA flow from its gene to the surrounding nucleoplasm is likely to be much more complex than generally assumed (see Smith et al., 1999). Defects in posttranscriptional RNA processing impair the full release and perturb the intranuclear fate of newly made RNA species (Custódio et al., 1999; Johnson et al., 2000). In this context, a rate-limiting step in the metabolism of these tandemly repeated, non-coding C19MC pri-miRNA transcripts, such as splicing and/ or 3' end of formation, might therefore prevent their commitment to normal intranuclear paths, thus explaining such a high local accumulation of pri-miRNA species. Their retention near transcription sites would then facilitate their correct processing by Microprocessor, as suggested for ectopically expressed
pri-miRNAs (Ballarino et al., 2009; Pawlicki and Steitz, 2008). We do not exclude the possibility that C19MC pri-miRNA processing can also be completed elsewhere, as evidenced by the detection of some DGCR8-bound nucleoplasmic pri-miRNAs (Figs 1, 2; supplementary material Fig. S2 and Movie 1).

FRAP analysis of GFP-Drosha and GFP-DGCR8 shows that the fluorescence recoveries of both proteins are dissociationdominated and that GFP-DGCR8 has a longer residence time at transcription sites than Drosha (Fig. 6A-C). One possibility is that Drosha and DGCR8 are recruited onto C19MC pri-miRNA separately. However, FCCS experiments demonstrate that DGCR8-Drosha complexes exist in the nucleoplasm even in the absence of transcription, arguing instead for the existence of pre-formed Microprocessor (Fig. 7). The Drosha non-interacting GFP-DGCR8(1-692) mutant is faithfully targeted to C19MC, with dynamics similar to those of wild-type GFP-DGCR8. Although we cannot exclude that this mutant is recruited to C19MC via its trimerization with endogenous DGCR8 (Faller et al., 2007; Han et al., 2004), we favor the explanation that DGCR8 has the potential to recognize C19MC pri-miRNAs in vivo in the absence of Drosha. This interpretation accounts for the substantial amounts of DGCR8 found at C19MC in Droshadepleted cells (Fig. 4) and is in agreement with in vitro data showing that purified DGCR8 interacts with pri-miRNA directly and specifically (Han et al., 2006; Lee et al., 2006; Yeom et al., 2006).

Taking these observations together, we propose the three-step model illustrated in Fig. 8. First, DGCR8 and Drosha are preferentially recruited as a pre-formed complex onto unspliced pri-miRNAs deposited near C19MC transcription sites (Fig. 8, Ia). Second, upon pri-miRNA processing, shortly after or concomitant with Drosha-mediated endonucleolytic cleavages, Microprocessor undergoes conformational changes (Fig. 8, IIb) that trigger the release of Drosha, which interacts more transiently with pri-miRNAs, consistent with its faster recovery at transcription sites. Third, DGCR8 remains associated with partially processed C19MC pri-miRNA species in the case of 'abortive processing', for example, Drosha dissociates before introducing cleavages (Fig. 8, IIIc), or through its ability to anchor the single-stranded RNA-double-stranded RNA junction (Fig. 8, IIId). We speculate that DGCR8 might also bind to

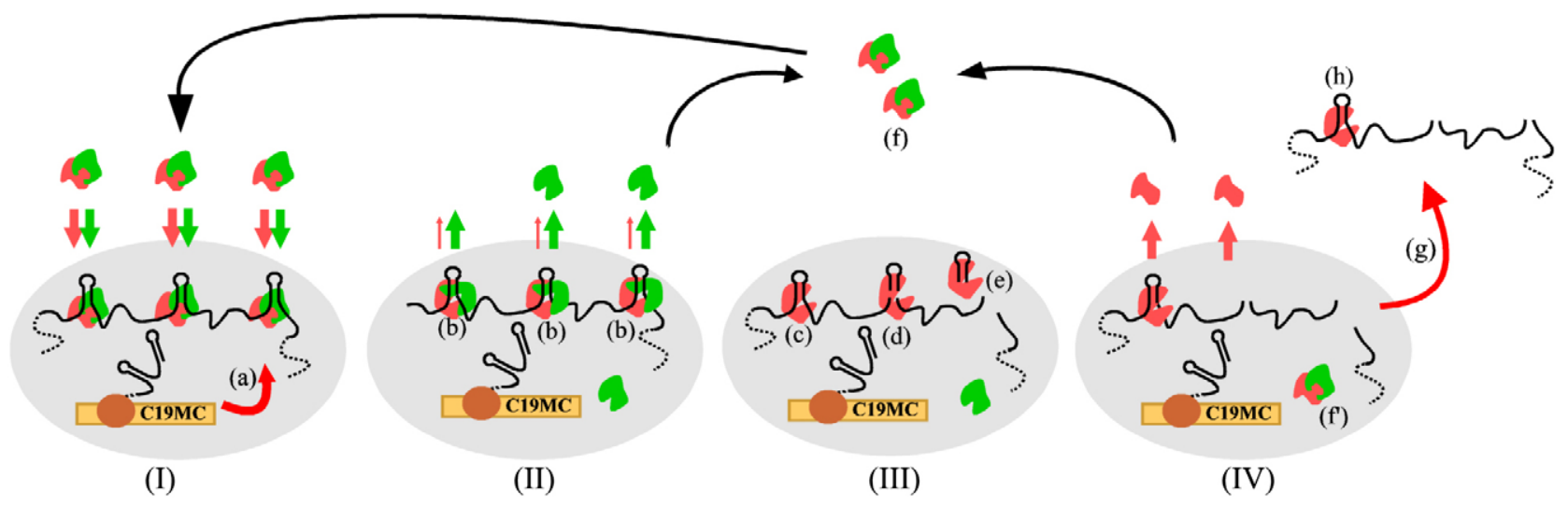

Fig. 8. Model for nuclear pri-miRNA processing at the C19MC locus. Recruitment of pre-formed Microprocessor onto unspliced pri-miRNAs close to C19MC transcription sites (a, gray area) is shown in I. Only three out of 46 C19MC pre-miRNAs (stem-loops) are depicted, with Drosha and DGCR8 represented by green and pink monomers, respectively. See Discussion for details of steps II and III. Reassembly, recycling and possible fates of pri-miRNAs are depicted in IV. 
processed pre-miRNAs before their commitment to the nuclear export pathway (Fig. 8, IIIe). This last step is followed by dissociation of DGCR8, Microprocessor reassembly elsewhere in the nucleoplasm (Fig. 8, IVf) and also most probably in the close vicinity of C19MC genes ('recycling Microprocessor'; Fig. 8, IVf). Some partially processed DGCR8-bound-pri-miRNAs, as well as pri-miRNAs lacking Microprocessor, escape transcription sites (Fig. 8, IVg) and could possibly be further processed in the nucleoplasm (Fig. 8, IVh), or simply be subjected to nuclear RNA decay. This model is supported by previous biochemical work emphasizing a crucial role for DGCR8 in pre-miRNA recognition as well as in the recruitment of Drosha (Han et al., 2006; Lee et al., 2006; Yeom et al., 2006). It also extends our knowledge of the mode of action of the Microprocessor complex by predicting conformational changes of the DGCR8-Drosha complex upon pri-miRNA processing. Additional work is now underway to decipher how Microprocessor is targeted to C19MC in relation to other gene processes and to elucidate the potential roles of additional trans-acting factors in the intranuclear fate of pri-miRNAs.

\section{Materials and Methods}

\section{Cell cultures, transfection, plasmids and antibodies}

JEG3 and HeLa cells were grown in DMEM (Gibco) supplemented with $10 \%$ fetal bovine serum and antibiotics, at $37{ }^{\circ} \mathrm{C}$ with $5 \% \mathrm{CO}_{2}$. Cells were transfected with the plasmids encoding GFP-fused proteins either using Lipofectamine 2000 (Invitrogen) according to the manufacturer's instructions or using $\mathrm{CaCl}_{2}$ as described (http:// www.lamondlab.com/pdf/CaPTf.pdf). RNAi experiments were performed as described (Bortolin-Cavaillé et al., 2009). The GFP-p54nrb plasmid was kindly provided by Archa H. Fox (University of Western Australia, Crawley, Australia). The cloning strategies for GFP-DGCR8, GFP-Drosha and mCherry-DGCR8 plasmids, as well as the primer sequences used for mutagenesis, are available upon request Antibodies and dilutions used were: rabbit $\alpha$-Drosha (Abcam, ab12286; 1:100 1:300), goat $\alpha$-DGCR8 (Santa Cruz Biotechnology, sc-48473; 1:200-1:500), mouse $4 \mathrm{H} 8$ antibody (Covance, MMS-128P-0100; 1:200), rabbit $\alpha$-H3K36me3 (Abcam, ab9050; 1:200), Alexa-Fluor-488-conjugated donkey anti-goat (Invitrogen, A11055 1:800), Alexa-Fluor-555-conjugated donkey anti-goat (Invitrogen. A21432; 1:800), Alexa-Fluor-488-conjugated donkey anti-rabbit (Invitrogen, A21206; 1:800), AlexaFluor-555-conjugated donkey anti-rabbit (Invitrogen, A31572; 1:800) and AlexaFluor-488-conjugated donkey anti-mouse (Invitrogen, A21202; 1:800)

\section{FISH microscopy}

RNA FISH experiments performed on in vitro cultivated cells were carried out as described (http://singerlab.aecom.yu.edu/protocols/). RNA FISH and immunostaining (immunofluorescence) experiments performed on $3-\mu \mathrm{m}$ paraffinembedded placenta sections were carried out as described (http://www.abcam $\mathrm{com} / \mathrm{ps} / \mathrm{pdf} /$ protocols/ihc_p.pdf), with the use of Tris-EDTA pH 9.0. Details for RNA FISH-immunofluorescence and RNA-DNA FISH experiments and the sequences of oligo-probes are available upon request. Images were captured with a CoolSnap ES camera (Roper Scientific) mounted on a Leica DMRB microscope (Leica Microsystems) with a $100 \times$ PL Fluotar objective (NA 1.3) using the Metavue software (Universal Imaging), or with a CoolSnap HQ camera (Princeton Instruments) mounted on an inverted IX-81 microscope (Olympus) with a $100 \times$ PL APO ON objective (NA 1.4) and the Metavue software. Most observations, including blind tests, were confirmed by at least two of the authors Images were slightly processed using Photoshop (Adobe) and ImageJ (NIH, Bethesda, MD). Use of additional filters and/or enhanced contrast is indicated in the figure legends.

\section{Fluorescence signal quantification methods}

The total fluorescence intensity of each dot was converted into the number of molecules of hybridized probes by using a published calibration procedure (Femino et al., 1998) modified as described below. Cy3-labeled oligo-probes in $3 \mu 1$ of Moviol medium (concentrations ranging from $0-4 \mathrm{ng} / \mu \mathrm{l}$ ) were first mounted between slides and coverslips coated with FITC-labeled beads. This allowed us to experimentally measure the distance $(d)$ between them and thus determine the volume of a voxel ( $\mathrm{pixel}^{2} \times d$ ). The concentration of oligo-probes (in ng/ $\mu \mathrm{l}$ ) was then converted into the number of oligo-probes per voxel. For each probe solution, three single-plane images were taken under the same conditions as the hybridized cells and the average gray level per pixel quantified using the ImageJ software. The calibration curve, obtained by plotting the gray level per pixel against the number of oligo-probes per voxel, was used to determine the number of hybridized oligo-probes within each nucleoplasmic RNA dot-like FISH signal. To quantify fluorescence intensity in each dot, digital images from a series of 18-30 focal plane were acquired in $0.18-\mu \mathrm{m}$ steps through hybridized nuclei. Planes were projected using the maximum-intensity method, and fluorescence dot quantification was performed using the Imaris software (Bitplane, Zurich, Switzerland). For twodimensional quantification of Microprocessor immunofluorescence signals, total fluorescence intensities of Drosha or DGCR8 immunofluorescence signals at transcription sites $(\mathrm{C} 19 \mathrm{MC})$ or at randomly chosen equivalent nucleoplasmic areas (Np) were measured on a single focal plane using the ImageJ software. These values were corrected by subtracting total fluorescence intensity of equivalent cytoplasmic areas used as background.

\section{GFP-Microprocessor, FRAP and FCCS}

Cells were plated on a Labtek II cell culture plate (Nunc) and analyzed 24 hours after transfection at $37^{\circ} \mathrm{C}$ and $5 \% \mathrm{CO}_{2}$ in OPTIMEM (Gibco). The Leica DMRB microscope (Leica Microsystems) with a $100 \times$ PL Fluotar objective (NA 1.3), or the DU888EC00 camera (Andor) mounted on an Olympus IX 81 microscope with a 100× Plan Apo objective (NA 1.4) were used for GFP-fused protein acquisitions. For FRAP experiments, a conventional inverted microscope (DMI6000B, Leica Microsystems) coupled to a FRAP 3D head (patent licensing from Curie InstituteRoper Scientific) and a DPSSL $473 \mathrm{~nm} 25 \mathrm{~mW}$ laser (Cobolt) was used. The microscope was equipped with a heated stage covered with an incubation system including $\mathrm{CO}_{2}$ and temperature controllers. Transcription sites were bleached with the laser, using a $3.75-\mu \mathrm{m}$ diameter circular region (bleaching time, 12 milliseconds). Beam guiding was performed using a FRAP 3D head coupled to the microscope through the epifluorescence port. The beam was focused through a high numerical-aperture objective lens $(100 \times$, HCS Plan Apo, NA 1.4 , oil immersion). Recoveries were recorded using a Xe-Hg light and a CoolSnap HQ2 cooled CCD camera (Roper Scientific, Princeton Instruments). Steering was performed using the Metamorph software. For transcription-site recovery, stacks of five images $1.2 \mu \mathrm{m}$ apart were collected every 10 to 180 seconds. For nucleoplasmic recovery, a live replay was performed on a single plane. For image analysis, fluorescence intensities were measured either in a small circle placed around the most intense area of the transcription site or in the nucleoplasm, outside the transcription-site area. Fluorescence intensities were normalized as described (Phair and Misteli, 2000). The recovery curves of 10-13 nuclei were averaged and fitted with the diffusion-uncoupled Sprague-McNally model (Sprague et al., 2004). The best-fitting curve to the experimental data was obtained by the least-squares fitting method.

FCCS was performed on an inverted Olympus microscope with a $63 \times$ water objective (NA 1.3) equipped with the MicroTime 200 FCCS extension from PicoQuant (Germany). GFP was excited using the $470 \mathrm{~nm}$ laser line of a pulseddiode laser that allowed for cross-talk correction using background or decay filters for Cherry or GFP, computed with the SymphoTime software from PicoQuant Cherry was excited with the $561 \mathrm{~nm}$ laser line of a DPSS diode laser (Coherent). The detection window for GFP fluorescence was $500-550 \mathrm{~nm}$ and $573-613 \mathrm{~nm}$ for Cherry fluorescence. Auto- and cross-correlation functions including cross-talk filtering were computed using the SymphoTime software. The relative crosscorrelation between the two channels was calculated on the basis of the auto- and cross-correlation amplitudes according to the ratio $G=G$ (cross) $/ \sqrt{ }[G$ (green channel $) \times G($ red channel) $]$ and expressed as a percentage. For each condition, at least 15 cells were measured. However, as FCCS relies on equal protein concentrations, only cells in which numbers of Drosha and DGCR8 molecules in the focal volume differed by no more than fivefold were considered for analysis. Failure to fit the correlation functions with diffusion models was most probably due to the fact that both Drosha and DGCR8 showed autocorrelation functions containing multiple components, including a very slow one with a correlation time of more than 100 milliseconds.

\section{Acknowledgements}

We thank Emmanuel Käs for careful reading of the manuscript. We are indebted to Corinne Lorenzo (LMBCP-CNRS) for helpful advice for FRAP and to Martine Yerle (INRA, Castanet, France) for chromosome painting experiments. We are very grateful to Nicolas Audugé and Maité Coppey (Institut Jacques Monod, Paris, France) for their help with FCCS, Guillaume Canal for help with the R software and Laurence Lamant for the generous gift of human placenta tissues. The probes for RNA FISH were synthesized by Jean-Marc Escudier (Modified Nucleic Acids Group, University Paul Sabatier, Toulouse, France).

\section{Funding}

This work was supported by grants from the European Molecular Biology Organization (EMBO Young Investigator Programme) to J.C.; the Agence National pour la Recherche (ANR-ImpmiR) to J.C.; 
the Association pour la Recherche sur le Cancer (ARC) to J.C.; the Ligue Regionale contre le Cancer to J.C.; and a PhD fellowship from the RegRNA program of the Paul Sabatier University (AOCS 20092010) to C.B.

\section{Supplementary material available online at}

http://jcs.biologists.org/lookup/suppl/doi:10.1242/jcs.100354/-/DC1

\section{References}

Bacia, K., Kim, S. A. and Schwille, P. (2006). Fluorescence cross-correlation spectroscopy in living cells. Nat. Methods 3, 83-89.

Ballarino, M., Pagano, F., Girardi, E., Morlando, M., Cacchiarelli, D., Marchioni, M., Proudfoot, N. J. and Bozzoni, I. (2009). Coupled RNA processing and transcription of intergenic primary microRNAs. Mol. Cell. Biol. 29, 5632-5638.

Bentwich, I., Avniel, A., Karov, Y., Aharonov, R., Gilad, S., Barad, O., Barzilai, A., Einat, P., Einav, U., Meiri, E. et al. (2005). Identification of hundreds of conserved and nonconserved human microRNAs. Nat. Genet. 37, 766-770.

Bortolin-Cavaillé, M. L., Dance, M., Weber, M. and Cavaillé, J. (2009). C19MC microRNAs are processed from introns of large Pol-II, non-protein-coding transcripts. Nucleic Acids Res. 37, 3464-3473.

Bushati, N. and Cohen, S. M. (2007). microRNA functions. Annu. Rev. Cell Dev. Biol. 23, 175-205.

Custódio, N., Carmo-Fonseca, M., Geraghty, F., Pereira, H. S., Grosveld, F. and Antoniou, M. (1999). Inefficient processing impairs release of RNA from the site of transcription. EMBO J. 18, 2855-2866.

Darzacq, X., Yao, J., Larson, D. R., Causse, S. Z., Bosanac, L., de Turris, V., Ruda, V. M., Lionnet, T., Zenklusen, D., Guglielmi, B. et al. (2009). Imaging transcription in living cells. Annu. Rev. Biophys. 38, 173-196.

Davis, B. N., Hilyard, A. C., Lagna, G. and Hata, A. (2008). SMAD proteins control DROSHA-mediated microRNA maturation. Nature 454, 56-61.

Denli, A. M., Tops, B. B., Plasterk, R. H., Ketting, R. F. and Hannon, G. J. (2004). Processing of primary microRNAs by the Microprocessor complex. Nature 432, 231235.

Faller, M., Matsunaga, M., Yin, S., Loo, J. A. and Guo, F. (2007). Heme is involved in microRNA processing. Nat. Struct. Mol. Biol. 14, 23-29.

Fang, Y. and Spector, D. L. (2007). Identification of nuclear dicing bodies containing proteins for microRNA biogenesis in living Arabidopsis plants. Curr. Biol. 17, 818823.

Femino, A. M., Fay, F. S., Fogarty, K. and Singer, R. H. (1998). Visualization of single RNA transcripts in situ. Science 280, 585-590.

Filipowicz, W., Bhattacharyya, S. N. and Sonenberg, N. (2008). Mechanisms of posttranscriptional regulation by microRNAs: are the answers in sight? Nat. Rev. Genet. $\mathbf{9}$, 102-114

Fujioka, Y., Utsumi, M., Ohba, Y. and Watanabe, Y. (2007). Location of a possible miRNA processing site in $\mathrm{SmD} 3 / \mathrm{SmB}$ nuclear bodies in Arabidopsis. Plant Cell Physiol. 48, 1243-1253.

Gregory, R. I., Yan, K. P., Amuthan, G., Chendrimada, T., Doratotaj, B., Cooch, N. and Shiekhattar, R. (2004). The Microprocessor complex mediates the genesis of microRNAs. Nature 432, 235-240.

Guil, S. and Cáceres, J. F. (2007). The multifunctional RNA-binding protein hnRNP A1 is required for processing of miR-18a. Nat. Struct. Mol. Biol. 14, 591-596.

Han, J., Lee, Y., Yeom, K. H., Kim, Y. K., Jin, H. and Kim, V. N. (2004). The Drosha-DGCR8 complex in primary microRNA processing. Genes Dev. 18, 30163027

Han, J., Lee, Y., Yeom, K. H., Nam, J. W., Heo, I., Rhee, J. K., Sohn, S. Y., Cho, Y., Zhang, B. T. and Kim, V. N. (2006). Molecular basis for the recognition of primary microRNAs by the Drosha-DGCR8 complex. Cell 125, 887-901.

Han, J., Pedersen, J. S., Kwon, S. C., Belair, C. D., Kim, Y. K., Yeom, K. H., Yang, W. Y., Haussler, D., Blelloch, R. and Kim, V. N. (2009). Posttranscriptional crossregulation between Drosha and DGCR8. Cell 136, 75-84.

Johnson, C., Primorac, D., McKinstry, M., McNeil, J., Rowe, D. and Lawrence, J. B. (2000). Tracking COL1A1 RNA in osteogenesis imperfecta. splice-defective transcripts initiate transport from the gene but are retained within the SC35 domain. J. Cell Biol. 150, 417-432.

Kataoka, N., Fujita, M. and Ohno, M. (2009). Functional association of the Microprocessor complex with the spliceosome. Mol. Cell. Biol. 29, 3243-3254.

Kim, V. N., Han, J. and Siomi, M. C. (2009). Biogenesis of small RNAs in animals. Nat. Rev. Mol. Cell Biol. 10, 126-139.

Kim, Y. K. and Kim, V. N. (2007). Processing of intronic microRNAs. EMBO J. 26, 775-783.
Landthaler, M., Yalcin, A. and Tuschl, T. (2004). The human DiGeorge syndrome critical region gene 8 and Its D. melanogaster homolog are required for miRNA biogenesis. Curr. Biol. 14, 2162-2167.

Lawrence, J. B., Singer, R. H. and Marselle, L. M. (1989). Highly localized tracks of specific transcripts within interphase nuclei visualized by in situ hybridization. Cell 57, 493-502.

Lee, Y., Han, J., Yeom, K. H., Jin, H. and Kim, V. N. (2006). Drosha in primary microRNA processing. Cold Spring Harb. Symp. Quant. Biol. 71, 51-57.

Leung, K. N., Vallero, R. O., DuBose, A. J., Resnick, J. L. and LaSalle, J. M. (2009). Imprinting regulates mammalian snoRNA-encoding chromatin decondensation and neuronal nucleolar size. Hum. Mol. Genet. 18, 4227-4238.

Michlewski, G., Guil, S., Semple, C. A. and Cáceres, J. F. (2008). Posttranscriptional regulation of miRNAs harboring conserved terminal loops. Mol. Cell 32, 383-393.

Morlando, M., Ballarino, M., Gromak, N., Pagano, F., Bozzoni, I. and Proudfoot, N. J. (2008). Primary microRNA transcripts are processed co-transcriptionally. Nat. Struct. Mol. Biol. 15, 902-909.

Noguer-Dance, M., Abu-Amero, S., Al-Khtib, M., Lefèvre, A., Coullin, P., Moore, G. E. and Cavaillé, J. (2010). The primate-specific microRNA gene cluster (C19MC) is imprinted in the placenta. Hum. Mol. Genet. 19, 3566-3582.

Pawlicki, J. M. and Steitz, J. A. (2008). Primary microRNA transcript retention at sites of transcription leads to enhanced microRNA production. J. Cell Biol. 182, 61-76.

Pawlicki, J. M. and Steitz, J. A. (2009). Subnuclear compartmentalization of transiently expressed polyadenylated pri-microRNAs: processing at transcription sites or accumulation in SC35 foci. Cell Cycle 8, 345-356.

Pawlicki, J. M. and Steitz, J. A. (2010). Nuclear networking fashions pre-messenger RNA and primary microRNA transcripts for function. Trends Cell Biol. 20, 52-61.

Phair, R. D. and Misteli, T. (2000). High mobility of proteins in the mammalian cell nucleus. Nature 404, 604-609.

Politz, J. C., Zhang, F. and Pederson, T. (2006). MicroRNA-206 colocalizes with ribosome-rich regions in both the nucleolus and cytoplasm of rat myogenic cells. Proc. Natl. Acad. Sci. USA 103, 18957-18962.

Politz, J. C., Hogan, E. M. and Pederson, T. (2009). MicroRNAs with a nucleolar location. RNA 15, 1705-1715.

Royo, H., Basyuk, E., Marty, V., Marques, M., Bertrand, E. and Cavaillé, J. (2007). Bsr, a nuclear-retained RNA with monoallelic expression. Mol. Biol. Cell 18, $2817-$ 2827.

Shiohama, A., Sasaki, T., Noda, S., Minoshima, S. and Shimizu, N. (2007). Nucleolar localization of DGCR8 and identification of eleven DGCR8-associated proteins. Exp. Cell Res. 313, 4196-4207.

Shopland, L. S., Johnson, C. V. and Lawrence, J. B. (2002). Evidence that all SC-35 domains contain mRNAs and that transcripts can be structurally constrained within these domains. J. Struct. Biol. 140, 131-139.

Siomi, H. and Siomi, M. C. (2010). Posttranscriptional regulation of microRNA biogenesis in animals. Mol. Cell 38, 323-332.

Smith, K. P., Moen, P. T., Wydner, K. L., Coleman, J. R. and Lawrence, J. B. (1999). Processing of endogenous pre-mRNAs in association with SC-35 domains is gene specific. J. Cell Biol. 144, 617-629.

Song, L., Han, M. H., Lesicka, J. and Fedoroff, N. (2007). Arabidopsis primary microRNA processing proteins HYL1 and DCL1 define a nuclear body distinct from the Cajal body. Proc. Natl. Acad. Sci. USA 104, 5437-5442.

Sprague, B. L., Pego, R. L., Stavreva, D. A. and McNally, J. G. (2004). Analysis of binding reactions by fluorescence recovery after photobleaching. Biophys. J. 86, 3473-3495.

Suzuki, H. I., Yamagata, K., Sugimoto, K., Iwamoto, T., Kato, S. and Miyazono, K. (2009). Modulation of microRNA processing by p53. Nature 460, 529-533.

Thomson, J. M., Newman, M., Parker, J. S., Morin-Kensicki, E. M., Wright, T. and Hammond, S. M. (2006). Extensive post-transcriptional regulation of microRNAs and its implications for cancer. Genes Dev. 20, 2202-2207.

Viswanathan, S. R., Daley, G. Q. and Gregory, R. I. (2008). Selective blockade of microRNA processing by Lin28. Science 320, 97-100.

Vitali, P., Royo, H., Marty, V., Bortolin-Cavaillé, M. L. and Cavaillé, J. (2010). Long nuclear-retained non-coding RNAs and allele-specific higher-order chromatin organization at imprinted snoRNA gene arrays. J. Cell Sci. 123, 70-83.

Wu, H., Xu, H., Miraglia, L. J. and Crooke, S. T. (2000). Human RNase III is a 160kDa protein involved in preribosomal RNA processing. J. Biol. Chem. 275, 3695736965.

Yamagata, K., Fujiyama, S., Ito, S., Ueda, T., Murata, T., Naitou, M., Takeyama, K., Minami, Y., O'Malley, B. W. and Kato, S. (2009). Maturation of microRNA is hormonally regulated by a nuclear receptor. Mol. Cell 36, 340-347.

Yeom, K. H., Lee, Y., Han, J., Suh, M. R. and Kim, V. N. (2006). Characterization of DGCR8/Pasha, the essential cofactor for Drosha in primary miRNA processing. Nucleic Acids Res. 34, 4622-4629. 